UB-HET-02-01

January 2002

\title{
Physics at Future Hadron Colliders
}

\author{
Convenors: U. Baur (Buffalo), R. Brock (MSU), J. Parsons (Columbia) \\ Subgroup Convenors: M. Albrow (FNAL), D. Denisov (FNAL), T. Han \\ (Wisconsin), A. Kotwal (Duke), F. Olness (SMU), J. Qian (Michigan)
}

Working Group: S. Belyaev (FSU), M. Bosman (Barcelona), G. Brooijmans (FNAL), I. Gaines (FNAL), S. Godfrey (Carleton), J.B. Hansen (CERN), J. Hauser (UCLA), U. Heintz (BU), I. Hinchliffe (LBL), C. Kao (Oklahoma), G. Landsberg (Brown), F. Maltoni (UIUC), C. Oleari (Wisconsin), C. Pagliarone (Pisa), F. Paige (BNL), T. Plehn (Wisconsin), D. Rainwater (FNAL), L. Reina (FSU), T. Rizzo (SLAC), S. Su (Caltech), T. Tait (ANL), D. Wackeroth (Rochester), E. Vataga (Pisa), D. Zeppenfeld (Wisconsin)

(Dated: December 11, 2013)

\begin{abstract}
We discuss the physics opportunities and detector challenges at future hadron colliders. As guidelines for energies and luminosities we use the proposed luminosity and/or energy upgrade of the LHC (SLHC), and the Fermilab design of a Very Large Hadron Collider (VLHC). We illustrate the physics capabilities of future hadron colliders for a variety of new physics scenarios (supersymmetry, strong electroweak symmetry breaking, new gauge bosons, compositeness and extra dimensions). We also investigate the prospects of doing precision Higgs physics studies at such a machine, and list selected Standard Model physics rates.
\end{abstract}

\section{INTRODUCTION}

Particle physics experiments at the highest possible energies, with their unique ability to explore the uncharted territory at the energy frontier, provide exciting opportunities for great advances. For the past two decades hadron colliders, the CERN $p \bar{p}$ collider and the Fermilab Tevatron, have held the high energy frontier and have resulted in the discovery of the $W$ and $Z$-bosons, and the top quark. For at least the next half-decade, the Tevatron will continue discovery physics experiments at the energy frontier and in a few years $(\sim 2006)$, the LHC will become the highest energy accelerator by colliding proton beams at a center of mass energy of $\sqrt{s}=14 \mathrm{TeV}$. The LHC will provide excellent chances to discover the Higgs boson and new physics at the $\mathrm{TeV}$ scale. With the construction of the LHC and its detectors well under way, and in view of the substantial time needed for planning and construction of large scale high energy physics projects, it is timely to consider possibilities for upgrading the LHC and for constructing a new hadron collider capable of reaching energies of $\mathcal{O}(100 \mathrm{TeV})$. Such a machine should be viewed as post LHC and Linear Collider (LC) and would allow access to unprecedented energy scales breaking completely new ground.

For the LHC both a luminosity upgrade by a factor 10 to $\mathcal{L}=10^{35} \mathrm{~cm}^{-2} \mathrm{~s}^{-1}$ and an energy upgrade by a factor 2 to $\sqrt{s}=28 \mathrm{TeV}$ are being discussed (so-called SuperLHC (SLHC)). Doubling the center of mass energy of the LHC would require new magnets with a field strength of about $17 \mathrm{~T}$. Such magnets currently do not exist. In contrast, a gradual increase of the luminosity by up to a factor 10 by increasing the bunch intensity to the beam - beam limit, replacing the quadrupole magnets near the interaction point, and reducing the bunch spacing by a factor 2 to $12.5 \mathrm{~ns}$ appears to be technologically feasible and, if so, can probably be achieved within 5 years after the LHC begins operation. From the experimental point of view, an increase in the center of mass energy is easier to exploit than an increase in luminosity. Even with major upgrades, the performance of the LHC detectors ATLAS [1] and CMS [2] will be degraded at $\mathcal{L}=10^{35} \mathrm{~cm}^{-2} \mathrm{~s}^{-1}$, due to the increased occupancy, radiation and pile-up noise. The impact of both possibilities on the physics capabilities of the ATLAS detector are discussed in Ref. [3].

An example of a design study for a Very Large Hadron Collider (VLHC) capable of reaching energies of $\mathcal{O}(100 \mathrm{TeV})$ has recently been presented in Ref. [4]. The design discusses a representative two stage $p p$ collider. Both stages might be housed in a tunnel with a total circumference of $233 \mathrm{~km}$. The first phase (VLHC-I) would use $2 \mathrm{~T}$ super-ferric magnets in order to achieve a total center of mass energy of $\sqrt{s}=40 \mathrm{TeV}$ and a luminosity of $\mathcal{L}=10^{34} \mathrm{~cm}^{-2} \mathrm{~s}^{-1}$. If the technology of super-ferric magnets can be pushed to $3 \mathrm{~T}$ as anticipated [5], the center of mass energy of the VLHC-I could be increased to about $50 \mathrm{TeV}$. Stage 2 of the VLHC (VLHC-II) would make use of the VLHC-I ring as an injector and could reach energies between $125 \mathrm{TeV}$ and $200 \mathrm{TeV}$, depending on the field strength of the magnets [- $\left[\right.$. The luminosity for $\sqrt{s}=125 \mathrm{TeV}$ would be of order $\mathcal{L}=5.1 \cdot 10^{34} \mathrm{~cm}^{-2} \mathrm{~s}^{-1}$, and would gradually decrease to approximately $\mathcal{L}=2.1 \cdot 10^{34} \mathrm{~cm}^{-2} \mathrm{~s}^{-1}$ at $\sqrt{s}=200 \mathrm{TeV}$. At VLHC-I, the 


\section{Heavy W Production}

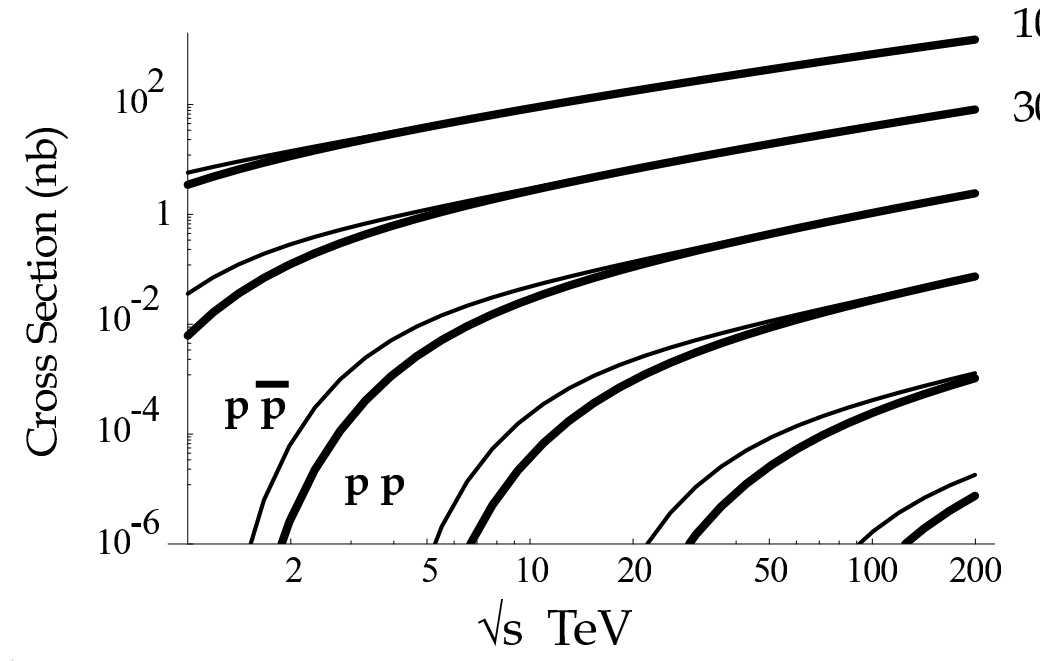

$\mathrm{M}_{\mathrm{W}}=$

$100 \mathrm{GeV}$

$300 \mathrm{GeV}$

$1 \mathrm{TeV}$

$3 \mathrm{TeV}$

$10 \mathrm{TeV}$

$30 \mathrm{TeV}$

FIG. 1: The total cross section for producing a heavy $W$ boson with SM like couplings in $p p$ and $p \bar{p}$ collisions as a function of the center of mass energy. For each value of $M_{W}$, the upper (thin) line gives the cross section for $p \bar{p} \rightarrow W^{ \pm}$, while the lower (thick) line shows the cross section $p p \rightarrow W^{ \pm}$.

number of interactions per bunch crossing would be similar to that at the LHC $(\approx 20)$. At VLHC-II, one would expect between 50 and 100 interactions per bunch crossing, depending of the center of mass energy. Luminosities up to $\mathcal{L}=10^{35} \mathrm{~cm}^{-2} \mathrm{~s}^{-1}$ at the highest center of mass energies considered are possible if the heat generated by synchrotron radiation [5] can be efficiently removed. Furthermore, the design can be modified to a single stage machine with $\sqrt{s}=150-200 \mathrm{TeV}$. In that scenario, a separate $5 \mathrm{TeV}$ injector would be built in a new $15 \mathrm{~km}$ circumference tunnel, which could also house a GigaZ $e^{+} e^{-}$collider [5].

In order to reach a center of mass energy of $200 \mathrm{TeV}$ in the VLHC tunnel, superconducting high field magnets with a field strength of about $11 \mathrm{~T}$ would be necessary. Such magnets could in principle also be installed in the Tevatron tunnel, or a site - filling tunnel at Fermilab, raising the possibility of a $p \bar{p}$ collider with a luminosity of $\mathcal{L}=10^{33} \mathrm{~cm}^{-2} \mathrm{~s}^{-1}$ and a center of mass energy of $\sqrt{s}=5.4 \mathrm{TeV}$ and $12 \mathrm{TeV}$, respectively. Studies have shown [6] that such a machine has a discovery potential for the Higgs boson and supersymmetry which is similar to that of the LHC. However, it would be very difficult to complete such a project before 2010, approximately 4 years after the LHC will commence operation.

With the Tevatron now running and the LHC on the horizon, it is appropriate to think ahead - to begin the investigation of the physics potential of and the detector requirements for hadron colliders with center of mass energies reaching the $100 \mathrm{TeV}$ region and/or luminosities which are up to a factor 10 larger than the LHC design luminosity. In this report we explore both aspects for a generic very large hadron collider (VLHC), selecting

- $\sqrt{s}=40 \mathrm{TeV}(\mathrm{VLHC}-\mathrm{I})$,

- $\sqrt{s}=100 \mathrm{TeV}$ (VLHC-II, low energy), and

- $\sqrt{s}=200 \mathrm{TeV}$ (VLHC-II, high energy)

as reference energies. Results will be presented for integrated luminosities in the range of $100 \mathrm{fb}^{-1} \mathrm{to}^{\mathrm{ab}} \mathrm{ab}^{-1}$. Whenever possible, we compare the physics reach of a VLHC with that of the SLHC. We shall assume that the VLHC is a $p p$ collider. For a given center of mass energy, the production cross section for a particle of mass $M$ in $p p$ and $p \bar{p}$ collisions is very similar due to the large sea - sea quark flux. The valence quark - anti-quark flux only contributes measurably to the cross section for $M>0.2 \sqrt{s}$. This is illustrated in Fig. 1 for a heavy $W$ boson with Standard Model (SM) like couplings. Since the luminosity of a $p \bar{p}$ collider is estimated to be about a factor 10 smaller than that of a $p p$ collider with the same center of mass energy, we shall only consider $p p$ collisions in the following.

The remainder of this report is organized as follows. In Section II we consider in some detail the physics opportunities at a VLHC. We discuss precision Higgs boson physics, the search for supersymmetry, and aspects of strong electroweak symmetry breaking relevant for future hadron colliders. We also discuss the search reach for new gauge bosons, compositeness of quarks and leptons, and extra dimensions. For completeness, we also 

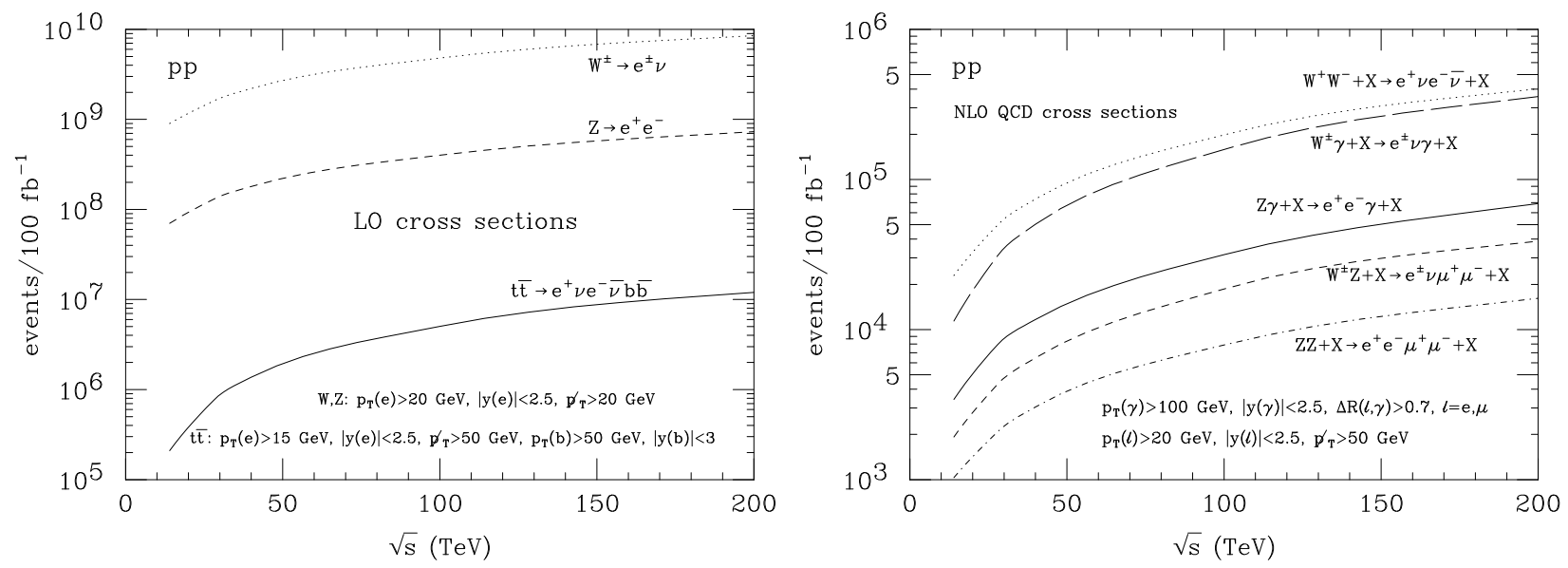

FIG. 2: Event rates in $p p$ collisions for selected SM processes as a function of $\sqrt{s}$. The cuts imposed are shown in the figure.

give results for selected SM cross sections. At the LHC and a linear $e^{+} e^{-}$collider, the search for the Higgs boson and precision Higgs boson physics provide well-motivated, specific "physics cases". Not surprisingly, this is not the case for a VLHC. Since such a machine will explore terra incognita, we can speculate about the physics to be discovered with such a facility, but cannot at present make the case that a particular discovery is likely to occur. In Section III we discuss the detector requirements for a VLHC and outline a R\&D program necessary in order to achieve the detector performance needed. Finally, in Section [V], we summarize our results and discuss future perspectives.

\section{PHYSICS POTENTIAL OF FUTURE HADRON COLLIDERS}

In this Section, we explore the physics opportunities at a VLHC. All results reported here are preliminary and represent only first steps towards a more complete analysis of the physics capabilities of such a machine. As noted above, a VLHC will come after at least two discovery machines (Tevatron and LHC) will have had considerable experience. We expect that the physics panorama will be different from what we face today. Therefore, it is important to note that the various scenarios considered often serve as place-holders for the physics menu of 15 - 20 years from now. We can be confident that pathways to this new physics will still pass through the need to be able to measure jets, leptons, photons and missing energy and have to function within radiation and rate environments which can be reliably predicted. However, the physics reactions chosen primarily act as illustrations of capabilities and indications of challenges which might be faced. They do not pretend to function as 2001 justifications for a 20?? VLHC or SLHC machine. The lead-time for design and construction will possibly prevent us from working within a realm of confident prediction of specific physics predictions. Whenever appropriate we compare the physics reach of the VLHC with that of the SLHC and that of linear $e^{+} e^{-}$colliders under consideration, such as Tesla [7], the NLC [8], the JLC [9] $(\sqrt{s}=500-1500 \mathrm{GeV}$, $\mathcal{L}=$ few $\left.\times 10^{34} \mathrm{~cm}^{-2} \mathrm{~s}^{-1}\right)$, or $\operatorname{CLIC}\left(\sqrt{s}=3-5 \mathrm{TeV}, \mathcal{L}=10^{35} \mathrm{~cm}^{-2} \mathrm{~s}^{-1}\right)$ 10, 11 .

\section{A. Standard Model Cross Sections}

By the time a VLHC-I would begin operation those parameters of the SM which are currently not well constrained, such as the top quark mass, $m_{t}$, the Higgs boson mass, $M_{H}$, or the gauge boson self-interactions, should be known very accurately from data collected at the LHC and/or a linear $e^{+} e^{-}$collider. The reason for building a VLHC therefore is not to test the SM but to directly search for new physics. However, many SM processes, such as top quark production, weak boson or weak boson pair production, are important sources of backgrounds for new physics processes. Accurate knowledge of the cross sections for SM processes, including higher order QCD and electroweak corrections is necessary.

In Fig. 2 we show expected event rates for an integrated luminosity of $100 \mathrm{fb}^{-1}$ for selected SM processes as a function of $\sqrt{s}$, taking into account minimal acceptance cuts listed on the figures for the observed final state particles. The cross sections of SM processes grow by about a factor $10-50$ as $\sqrt{s}$ is increased from $14 \mathrm{TeV}$ to 
$200 \mathrm{TeV}$. The left panel shows that $W \rightarrow e \nu$ and $\bar{t} t \rightarrow e^{+} \nu e^{-} \bar{\nu} \bar{b} b$ events are produced with a rate of about $1 \mathrm{kHz}$ and $1 \mathrm{~Hz}$ respectively for $\sqrt{s}=200 \mathrm{TeV}$. This may allow for indirect searches of new physics in rare decays, such as $t \rightarrow W Z b\left(\mathrm{SM}: \mathrm{BR}(t \rightarrow W Z b) \approx 2 \cdot 10^{-6}[12]\right)$. The right panel displays the diboson cross sections at NLO in QCD. QCD corrections enhance the diboson rates by a factor $1.3-2.8$ at LHC energies 13 , and by a factor $1.9-5.0$ at $\sqrt{s}=200 \mathrm{TeV}$. The size of the QCD corrections is smallest for $Z \gamma$ production, and largest for $W \gamma$ production. The large enhancement of the diboson cross sections is due to a logarithmic enhancement factor in the $q g$ and $\bar{q} g$ real emission subprocesses. The cross section for $Z Z$ production also includes the contribution from gluon fusion [14]. At LHC and VLHC energies, the $g g \rightarrow Z Z$ cross section is not negligible compared to the lowest order $\bar{q} q \rightarrow Z Z$ rate:

$$
\begin{aligned}
& \sigma(g g \rightarrow Z Z) \approx 0.13 \sigma(\bar{q} q \rightarrow Z Z) \text { for } \sqrt{s}=14 \mathrm{TeV} \\
& \sigma(g g \rightarrow Z Z) \approx 0.45 \sigma(\bar{q} q \rightarrow Z Z) \text { for } \sqrt{s}=200 \mathrm{TeV}
\end{aligned}
$$

The cross section for $g g \rightarrow W^{+} W^{-} 15$ is less than $10 \%$ of the lowest order $\bar{q} q \rightarrow W^{+} W^{-}$cross section over the entire $\sqrt{s}$ considered here, and is therefore not included in Fig. 22.

\section{B. Precision Higgs Boson Physics}

If the SM Higgs boson exists, it will be discovered either at the Tevatron [16] or the LHC [17]. The Tevatron will be able to find the Higgs boson if its mass is less than about $170 \mathrm{GeV}$, assuming an integrated luminosity of $30 \mathrm{fb}^{-1}$ can be achieved per experiment. In contrast, the LHC can find the SM Higgs boson [17] over the entire range from the present lower experimental limit of $M_{H}>114.1 \mathrm{GeV}$ [18] to the TeV region. From a global fit to the electroweak observables, one obtains a 95\% CL upper limit of $\bar{M}_{H}<196 \mathrm{GeV}$ from present data 19 . In the following we concentrate on a SM Higgs boson with mass in the range $114 \mathrm{GeV}<M_{H}<200 \mathrm{GeV}$. The discovery potential of Higgs bosons in the minimal supersymmetric SM at the LHC and VLHC is discussed in Refs. [20, 21] and [22], respectively.

\section{Measurement of Higgs boson properties at the LHC/SLHC and a Linear Collider}

Once the Higgs boson has been discovered, it will be important to measure its properties in order to test whether its coupling to fermions and gauge bosons is as predicted by the SM. Even more important will be the measurement of the Higgs boson self-coupling, $\lambda_{H H H}$, which partly determines the shape of the Higgs potential. In the SM, $\lambda_{H H H}=3 M_{H}^{2} / 2 v$, where $v=246 \mathrm{GeV}$ is the vacuum expectation value. An accurate test of this relation may reveal whether the minimal Higgs sector of the SM, or an extended sector which arises for example in supersymmetric theories, is realized.

With an integrated luminosity of $300 \mathrm{fb}^{-1}$, ATLAS and CMS combined are expected to measure $M_{H}$ with a precision of about $0.1 \%$ [21, 23]. One also hopes to determine the total width of the Higgs boson, $\Gamma_{H}$, and $\sigma \times \mathrm{Br}$ for $H \rightarrow \gamma \gamma, H \rightarrow Z Z^{(*)} \rightarrow 4$ leptons and $H \rightarrow W W^{(*)} \rightarrow \ell \nu \ell \nu$ with a precision of about 10\% [23], ratios of couplings to gauge bosons and fermions with an accuracy of $10-20 \%$ [24], and the $t \bar{t} H$ coupling, $y_{t t H}$, with a precision of $13 \%$ 25. Since many of these measurements at the LHC are statistically limited, one can hope to improve the precision which can be achieved by a factor 1.5 to 2 if the LHC energy could be doubled to $\sqrt{s}=28 \mathrm{TeV}$. As discussed in Sec. II B6, jet tagging and central jet veto, which are crucial for determining the Higgs boson couplings at the LHC [24], become less efficient if the luminosity is increased beyond $\mathcal{L}=10^{34} \mathrm{~cm}^{-2} \mathrm{~s}^{-1}$. The measurement of the Higgs boson couplings is therefore expected to profit only modestly (by at most a factor 2) from a luminosity upgrade of the LHC 26. If $115 \mathrm{GeV}<M_{H}<140 \mathrm{GeV}$, the $H \mu \mu$ coupling may be determined with a precision of about $15 \%$ at the LHC, assuming that the $t \bar{t} H$ and $b \bar{b} H$ couplings are SM-like [27]. In order to probe the Higgs boson self-coupling, Higgs pair production processes have to be studied. The cross sections of the $H$ pair signal processes at the LHC have been calculated in Ref. [28], however, a study of the feasibility of measuring $\lambda_{H H H}$ has not yet been carried out. Such a measurement may well be very difficult at the LHC.

While the LHC will make it possible to perform first, but still somewhat rough, measurements of the Higgs boson properties, a LC will provide the opportunity to carry out more precise measurements of the couplings of the Higgs boson to gauge bosons and the fermions which belong to the third generation $(\tau, b$ and $t)$ [29]. At a LC with $\sqrt{s}=500 \mathrm{GeV}$ and $500 \mathrm{fb}^{-1}$, the couplings of the Higgs boson to $b$-quarks, $\tau$-leptons, photons, $W$-bosons and gluons can be measured with a precision of a few per cent [7, 8, 9. In addition, the top quark Yukawa coupling $y_{t t H}$ can be determined with a precision of about $5.5 \%(21 \%)$ at $\sqrt{s}=800 \mathrm{GeV}(\sqrt{s}=500 \mathrm{GeV})$ and with $1 \mathrm{ab}^{-1}$. A LC will also make it possible to perform a measurement of the $H \mu \mu$ and Higgs boson 


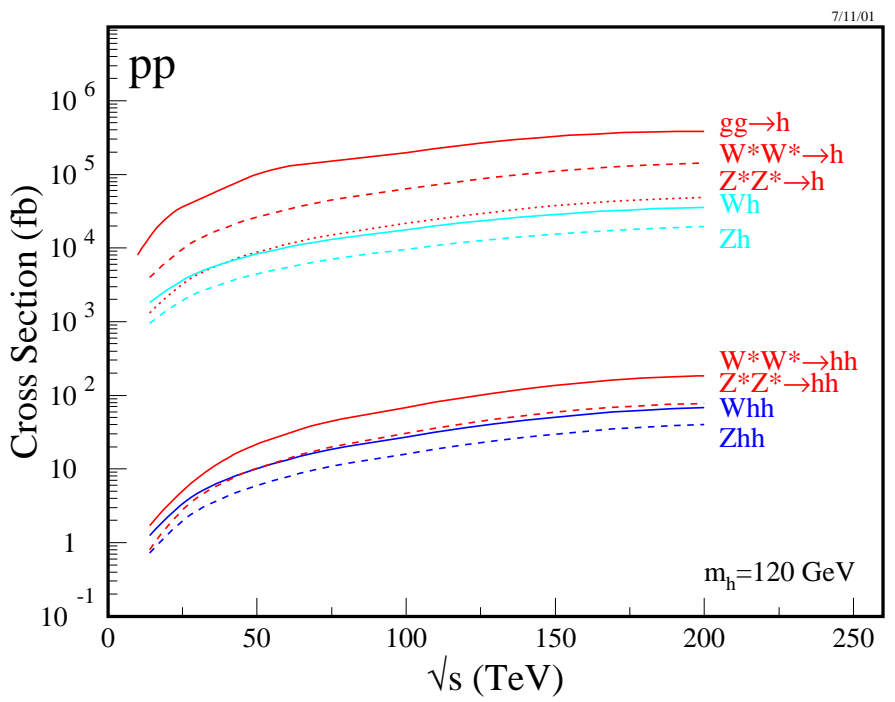

FIG. 3: Cross sections for various Higgs and Higgs pair production processes as a function of $\sqrt{s}$ for $p p$ collisions and $M_{H}=120 \mathrm{GeV}$.

self-coupling, although a multi-TeV collider with a luminosity of $\mathcal{L}=10^{35} \mathrm{~cm}^{-2} \mathrm{~s}^{-1}$ or more is required for a precise determination of these parameters. Recent studies have shown that the $H \mu \mu$ coupling can be determined with a precision of about $15 \%(4 \%)$ for $M_{H}=120 \mathrm{GeV}$ and an integrated luminosity of $1 \mathrm{ab}^{-1}\left(5 \mathrm{ab}^{-1}\right)$ at a LC operating at $\sqrt{s}=800 \mathrm{GeV}(\sqrt{s}=3 \mathrm{TeV})$ [30], and that the Higgs boson self-coupling can be measured with an accuracy of about $20 \%(7-8 \%)$ for $\sqrt{s}=500 \mathrm{GeV}(\sqrt{s}=3 \mathrm{TeV})$ and $5 \mathrm{ab}^{-1}$ [7, 8, 31]. The most natural place for extracting the muon Yukawa coupling of course is a muon collider [32], operating at energies around the Higgs boson mass. Assuming that the branching ratio for $H \rightarrow \bar{b} b$ can be determined to $2.5 \%$ at a LC, it should be possible to measure the $H \mu \mu$ coupling at such a machine with a precision of about $2 \%$ [33].

\section{Jet Tagging at VLHC Energies}

It is interesting to investigate whether an upgraded LHC or a VLHC offer a chance to improve the LC measurements of the Higgs boson couplings, in particular those of the $H \mu \mu$, the $t \bar{t} H$, and the $H H H$ couplings. The cross sections for Higgs production processes increase by about a factor $10-30$ from the LHC energy to the highest VLHC-II energy (see Fig. 3). In order to obtain information on the couplings of the Higgs boson at hadron colliders, it is crucial to make use of Higgs boson production via weak boson fusion (WBF), i.e. by separately observing $q q \rightarrow q q H$ and crossing related processes in which the Higgs boson is radiated off a $t$-channel $W$ or $Z$ [24. WBF events are characterized by two forward jets which are separated by a large rapidity gap. Forward jet tagging and a central jet veto thus are crucial to reduce the QCD background and to extract the WBF signal. The characteristics of the tagging jets in WBF at the LHC and at VLHC energies are shown in Fig. 1 for $M_{H}=120 \mathrm{GeV}$ and $M_{H}=800 \mathrm{GeV}$. It should be noted that very similar results are obtained if the Higgs boson is replaced by another massive (possibly composite) particle of equal mass as long as it couples to $W$ and $Z$ bosons and is produced via WBF. Such states frequently occur in models of dynamical symmetry breaking [35]. Figure ta shows the normalized differential cross section as a function of $|y|^{\max }=\max \left(\left|y\left(j_{1}\right)\right|,\left|y\left(j_{2}\right)\right|\right)$, where $y\left(j_{1}\right)$ and $y\left(j_{2}\right)$ are the rapidities of the tagging jets. Figure $\llbracket \mathrm{b}$ displays the normalized differential cross section as a function of the rapidity difference of the two jets, $\Delta y=\left|y\left(j_{1}\right)-y\left(j_{2}\right)\right|$. At the LHC, the $|y|^{\text {max }}$ distribution peaks at $|y|^{\text {max }} \approx 3-3.5$. With increasing machine energy, the peak moves to higher values of rapidities and broadens. For $\sqrt{s}=200 \mathrm{TeV}$, the maximum occurs at $|y|^{\text {max }} \approx 5.5$ with a long tail extending to values larger than 7 . The $\Delta y$ distribution peaks at $\Delta y \approx 4-4.5$ for a light Higgs boson for the energies considered. For a heavy state produced via WBF, the rapidity gap between the two jets is more pronounced and widens considerably with higher values of $\sqrt{s}$. For a $200 \mathrm{TeV}$ collider, the tagging jets are separated by typically $8-10$ units in rapidity for production of a heavy Higgs boson.

From Fig. 1 one concludes that it will be necessary to have a hadron calorimeter which covers the region out to $|y|=6-7$ in order to take full advantage of jet tagging in WBF. The vertical dotted line in Fig. 四 indicates the maximum rapidity for which the LHC detectors are efficient in detecting forward jets. If this cannot be improved, a large fraction of the WBF signal will be lost at the VLHC. 

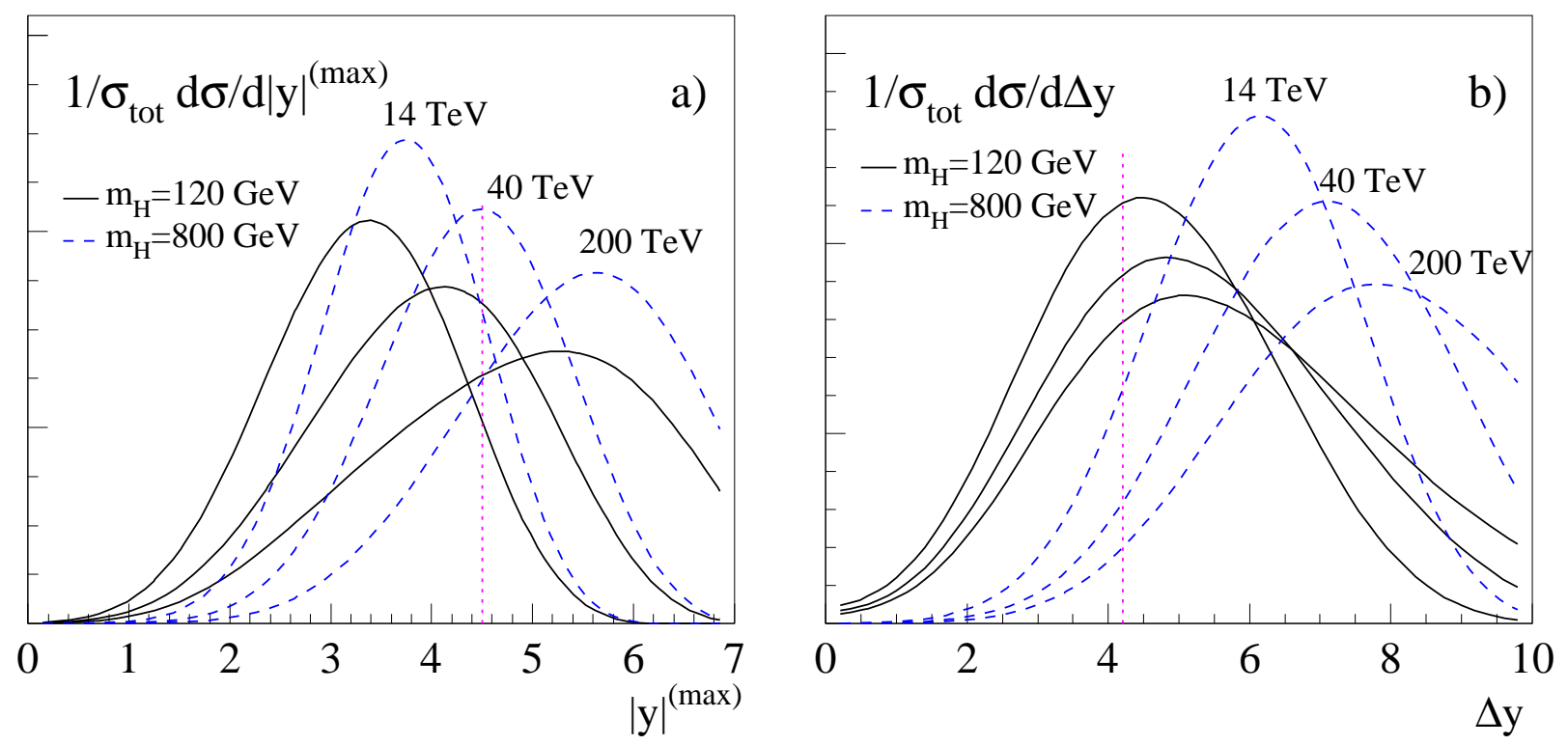

FIG. 4: a) Normalized maximum jet rapidity distribution and b) normalized rapidity difference distribution for the WBF signal for two values of $M_{H}$ and several center of mass energies. A $p_{T}\left(j_{1,2}\right)>30 \mathrm{GeV}$ cut is imposed on the tagging jets. The figure is taken from Ref. [34.

TABLE I: Significance of the $H \rightarrow \mu^{+} \mu^{-}$signal in WBF for $\int \mathcal{L} d t=300 \mathrm{fb}^{-1}$ at the LHC and VLHC (from Ref. [34).

\begin{tabular}{|c|c|c|c|}
\hline$M_{H}(\mathrm{GeV})$ & $\sqrt{s}=14 \mathrm{TeV}(\mathrm{LHC})$ & $\sqrt{s}=40 \mathrm{TeV}$ (VLHC-I) & $\sqrt{s}=200 \mathrm{TeV}$ (VLHC-II) \\
\hline 120 & 1.8 & 3.3 & 5.7 \\
130 & 1.7 & 3.2 & 5.3 \\
140 & 1.2 & 2.4 & 4.0 \\
\hline
\end{tabular}

\section{3. $H \rightarrow \mu^{+} \mu^{-}$and $H \rightarrow \tau^{+} \tau^{-}$at the $V L H C$}

The search for Higgs boson decays to muons is rate limited due to the extremely small $H \mu \mu$ coupling. For $g g \rightarrow H \rightarrow \mu^{+} \mu^{-}$, the processes $q \bar{q} \rightarrow \gamma, Z \rightarrow \mu^{+} \mu^{-}$constitute a large irreducible background. In WBF, on the other hand, jet tagging and central jet veto offer a handle to suppress the QCD $\mu^{+} \mu^{-} j j X$ background. The significance of the $H \rightarrow \mu^{+} \mu^{-}$signal in WBF at the LHC and the VLHC for several values of $M_{H}$ are shown in Table [ [34]. The following WBF selection cuts were imposed in Table [i:

$$
p_{T}\left(j_{1,2}\right)>20 \mathrm{GeV}, \quad \Delta R\left(j_{1}, j_{2}\right)>0.6, \quad\left|y\left(j_{1,2}\right)\right|<4.5, \quad\left|y\left(j_{1}\right)-y\left(j_{2}\right)\right|>4.2, \quad y\left(j_{1}\right) \cdot y\left(j_{2}\right)<0 .
$$

In order to reduce the $t \bar{t}, b \bar{b}$ and $Z j j$ backgrounds, an invariant mass cut on the two jet system was imposed:

$$
m(j j)>500 \mathrm{GeV} \quad(\mathrm{LHC}) \text { and } m(j j)>1000 \mathrm{GeV} \quad(\mathrm{VLHC}) .
$$

At VLHC-II, the decay $H \rightarrow \mu^{+} \mu^{-}$should be detectable for the Higgs boson masses studied, and, for an integrated luminosity of $1 \mathrm{ab}^{-1}$, it should be possible to measure the $H \mu \mu$ coupling with a precision similar to that which can be achieved at CLIC with $5 \mathrm{ab}^{-1}$ (assuming that the coupling of the Higgs boson to the $W$ and $Z$ bosons has been precisely measured before, eg. at a LC). If one includes the $g g \rightarrow H \rightarrow \mu^{+} \mu^{-}$channel, the precision improves by about a factor 1.5 27], provided that the $H g g$ coupling and higher order QCD corrections are known with sufficient precision. Further strengthening of the signal in WBF is possible if one assumes that the hadron calorimeter coverage extends to rapidities of $\left|y\left(j_{1,2}\right)\right| \approx 6-7$ instead of the value listed in (3).

In order to see whether the measurement of the Higgs boson couplings to the third generation fermions at the LC can be improved at a VLHC, the $H \rightarrow \tau^{+} \tau^{-}$channel in WBF was analyzed [36]. Imposing similar jet

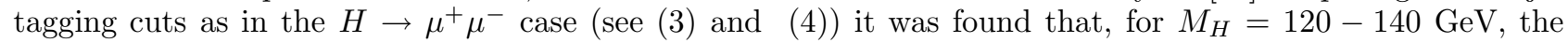
$H \tau \tau$ coupling can be measured at a VLHC with a statistical uncertainty of a few per cent for an integrated luminosity of $100 \mathrm{fb}^{-1}$. This is comparable with the precision expected at a LC. However, for $\sqrt{s}>100 \mathrm{TeV}$, 


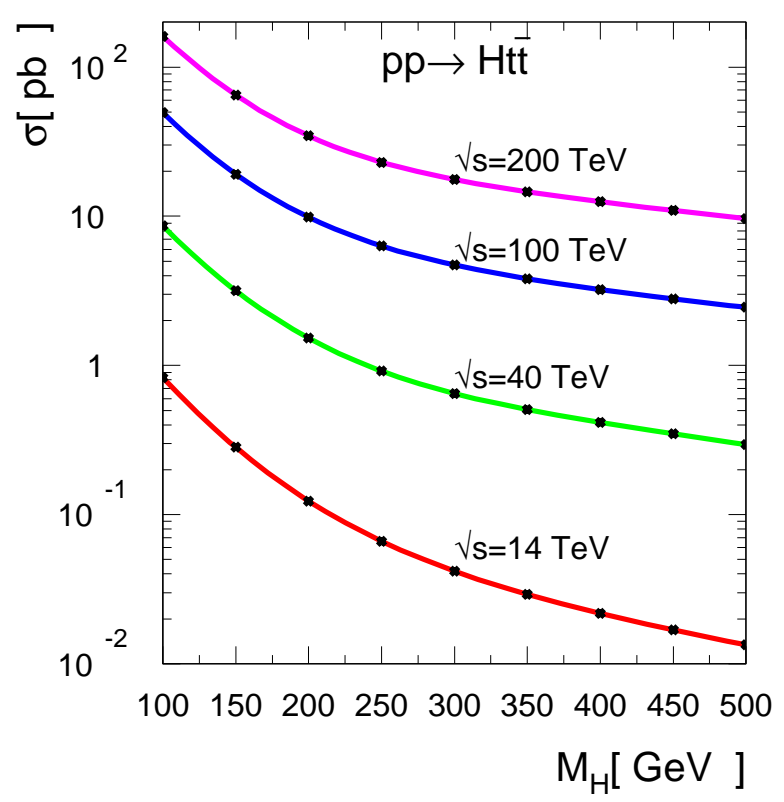

FIG. 5: The leading order cross section for $p p \rightarrow t \bar{t} H$ as a function of $M_{H}$ for various center of mass energies in the SM. The CTEQ4L set of parton distribution functions has been used to evaluate the cross section, and the renormalization and factorization scales have been set to $\mu=m_{t}+M_{H} / 2$ with $m_{t}=174 \mathrm{GeV}$.

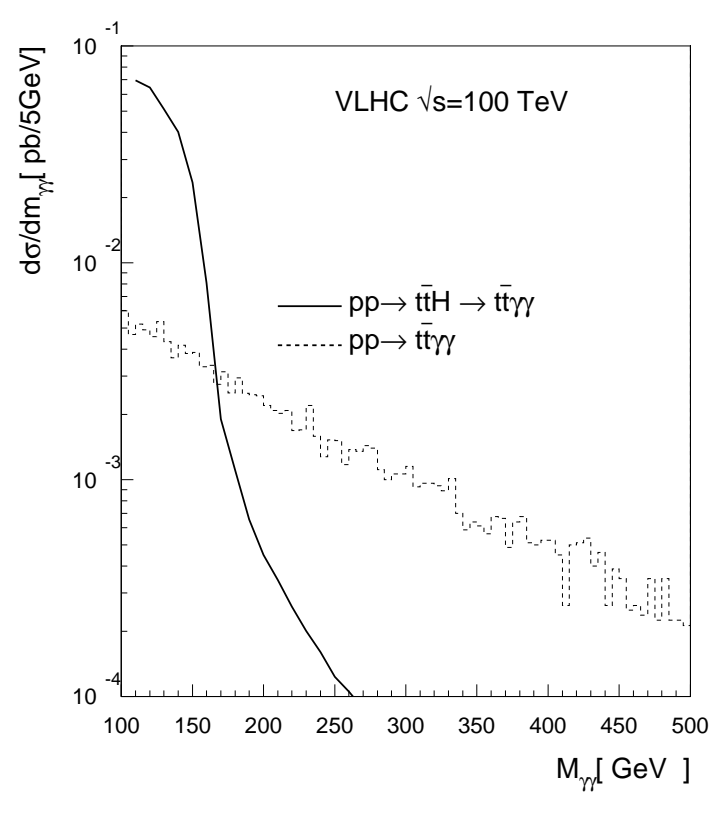

FIG. 6: Cross sections for the SM $\bar{t} t H(\rightarrow \gamma \gamma)$ signal (solid line) and the irreducible $t \bar{t} \gamma \gamma$ background (dashed histogram) at $\sqrt{s}=100 \mathrm{TeV}$. To simulate detector acceptance we require $p_{T}(\gamma)>25 \mathrm{GeV}$ and $|\eta(\gamma)|<3$.

gluon fusion becomes a pronounced source of $H+2$ jet events [37, and the clean separation of gluon fusion and WBF cross sections will require additional effort. More detailed studies are also necessary in order to assess the experimental systematic uncertainties.

\section{Measuring the Top Quark Yukawa Coupling at the VLHC in $t \bar{t} H$ Production}

The top quark Yukawa coupling at a hadron collider is most easily measured using $t \bar{t} H$ production. At the highest VLHC-II energies, the SM $t \bar{t} H$ cross section is a factor $100-1000$ larger than at the LHC 38 (see Fig. 5). The $t \bar{t} H$ production cross section at VLHC-II energies is sufficiently large that even rare decays such as $H \rightarrow \gamma \gamma$ can be observed in this mode. The SM $t \bar{t} H(\rightarrow \gamma \gamma)$ signal and the $t \bar{t} \gamma \gamma$ continuum background for $p p$ collisions at $\sqrt{s}=100 \mathrm{TeV}$ are shown in Fig. 6. For $M_{H}<150 \mathrm{GeV}$, the background is seen to be totally negligible. Taking into account realistic particle detection efficiencies and assuming that the $H \gamma \gamma$ coupling has been measured at the LHC or a linear collider, the top Yukawa coupling can be measured with an accuracy of about $7 \%$ for $M_{H}=130 \mathrm{GeV}$ and $\sqrt{s}=100 \mathrm{TeV}$ [38]. A similar analysis for $H \rightarrow \tau^{+} \tau^{-}$and $H \rightarrow \bar{b} b$ shows that $y_{t t H}$ can be determined with a precision of $3.5 \%$ and $1.5 \%$ in these channels. Of course this assumes that the $H \tau \tau$ and $H \bar{b} b$ couplings have been determined before, e.g. at the LHC $(H \tau \tau)$ or the LC $(H \tau \tau, H \bar{b} b)$. Alternatively, from the ratio of the cross sections in the $t \bar{t} \tau^{+} \tau^{-}$and $t \bar{t} b \bar{b}$ channels one can measure the strength of the $H \bar{b} b$ coupling. For $\sqrt{s}=200 \mathrm{TeV}$, the precision which one hopes to achieve for $y_{t t H}$ improves by a factor 1.5 to 2 over that obtained for $\sqrt{s}=100 \mathrm{TeV}$.

If the Higgs boson is in the mass range $140 \mathrm{GeV}<M_{H}<190 \mathrm{GeV}$, important information on the top quark Yukawa coupling can also be obtained from $p p \rightarrow t \bar{t} H\left(\rightarrow W^{+} W^{-}\right)$. One finds that the highest sensitivities are obtained from the $3 \ell+X$ final state. Figure 7 shows the precision for $y_{t t H}$ which one expects to achieve for $300 \mathrm{fb}^{-1}$ as a function of $M_{H}$ and several center of mass energies. The backgrounds from $t \bar{t} Z X, t \bar{t} W X, t \bar{t} W W$ and $t \bar{t} t \bar{t}$ production are taken into account in this analysis [39]. At the VLHC-I (VLHC-II), a measurement of $\delta y_{t t H} / y_{t t H} \approx 0.05-0.10\left(\delta y_{t t H} / y_{t t H} \approx 0.01-0.02\right)$ should be possible.

A VLHC will thus be able to considerably improve the measurement of the top quark Yukawa coupling over the precision which can be achieved at a linear $e^{+} e^{-}$collider (see Sec. II B 1 ). 


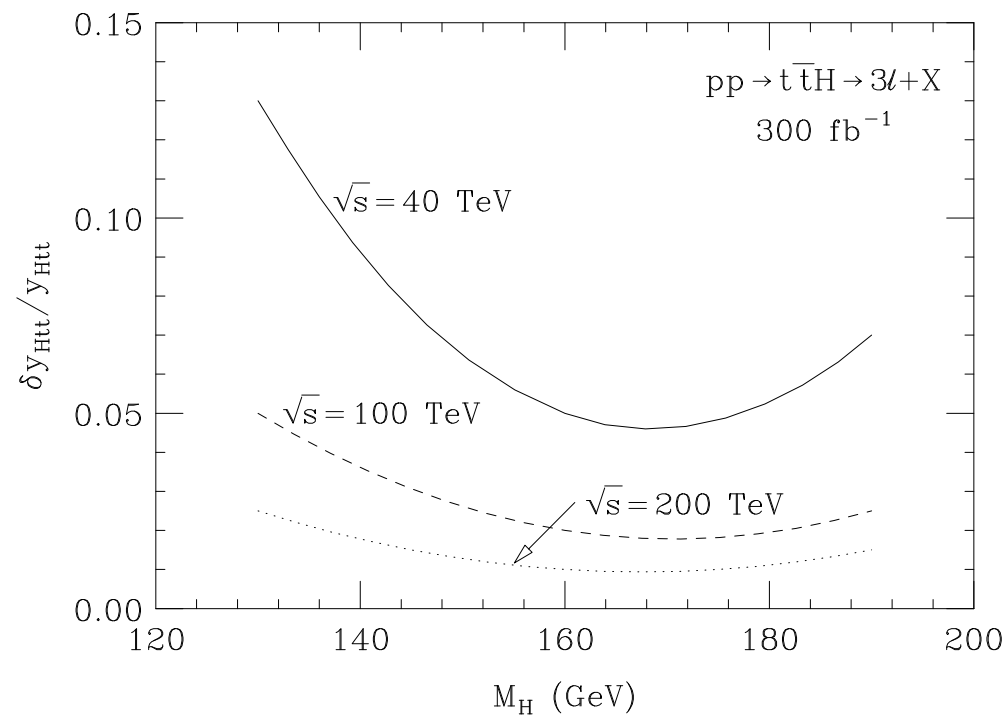

FIG. 7: The relative precision of the top quark Yukawa coupling as a function of the mass of the Higgs boson which can be obtained for an integrated luminosity of $300 \mathrm{fb}^{-1}$ in $p p \rightarrow \rightarrow t \bar{t} H\left(\rightarrow W^{+} W^{-}\right) \rightarrow 3 \ell+X$. To simulate detector response, the following cuts were imposed: $p_{T}(j)>30 \mathrm{GeV}, p_{T}(b)>30 \mathrm{GeV},|\eta(j)|<4.5,|\eta(b)|<2.5, p_{T}(\ell)>15 \mathrm{GeV}$, $|\eta(\ell)|<2.5$, and $\not p_{T}>50 \mathrm{GeV}$. In addition, events with $81 \mathrm{GeV}<m\left(\ell^{+} \ell^{-}\right)<101 \mathrm{GeV}$ are excluded. b-quarks (leptons) are assumed to be detected with an efficiency of $50 \%(85 \%)$.

\section{Can one probe the Higgs Potential at the VLHC?}

As mentioned before, Higgs pair production offers an opportunity to probe the Higgs boson self-coupling, $\lambda_{H H H}$. As can be seen from Fig. 3, the cross sections for Higgs pair production processes are a factor 100 to 1000 smaller than those for single Higgs production. Taking into account the small branching fractions for final states with manageable background, it is clear that it will be very difficult to measure $\lambda_{H H H}$ in a hadron collider environment. The most promising decay channel may be $H H \rightarrow \bar{b} b W^{+} W^{-} \rightarrow \bar{b} b \ell_{1} \ell_{2} p_{T}$. The branching ratio for this channel peaks at $\approx 0.8 \%$ for $M_{H} \approx 130 \mathrm{GeV}$. No detailed study of Higgs pair production at hadron colliders has been carried out so far. For $g g \rightarrow H H$, backgrounds from QCD induced processes are likely to be severe. Excellent $b$-tagging may help to reduce the background to an acceptable level. On the other hand, as in single Higgs boson production, the characteristics of WBF should make it possible to reduce the background to an acceptable level in $q q \rightarrow q q H H$. A simple estimate then shows that a minimum of several $\mathrm{ab}^{-1}$ is needed in order to be able to measure $\lambda_{H H}$ in this channel with a precision similar to that which has been predicted for a multi-TeV $e^{+} e^{-}$collider [31].

\section{Jet tagging and Central Jet Veto at $\mathcal{L}=10^{35} \mathrm{~cm}^{-2} \mathrm{~s}^{-1}$}

An integrated luminosity of several $\mathrm{ab}^{-1}$ can realistically only be achieved if the VLHC can be operated at a luminosity close to $\mathcal{L}=10^{35} \mathrm{~cm}^{-2} \mathrm{~s}^{-1}$. Jet tagging and central jet veto requirements, which are essential for WBF processes such as $q q \rightarrow q q H H$, are expected to become less useful with increasing luminosity as the pile-up of additional events can cause jets to appear in these regions and degrade the jet measurements. This was studied in detail in Ref. 30 for the SLHC. The results are summarized in Figs. 8 and 9. For the LHC design luminosity of $\mathcal{L}=10^{34} \mathrm{~cm}^{-2} \mathrm{~s}^{-1}$, the background from fake forward jets is small for $E(j)>300 \mathrm{GeV}$ and a jet veto cut of $p_{T}(j)<30 \mathrm{GeV}$ is feasible. For $\mathcal{L}=10^{35} \mathrm{~cm}^{-2} \mathrm{~s}^{-1}$, the minimum jet energy has to be increased beyond $E(j) \approx 1 \mathrm{TeV}$, and additional strategies would probably have to be employed in order to keep the background from fake forward jets manageable. In addition, the jet veto transverse momentum threshold has to be raised to $70-100 \mathrm{GeV}$. For smaller values, the probability for fake central jets from event pile-up is essentially $100 \%$. Combined, the increased minimum energy of the tagging jets, and the higher jet veto $p_{T}$ threshold compensate the increased number of events at the higher luminosity [3]. Similar conclusions are expected to hold for VLHC energies. 


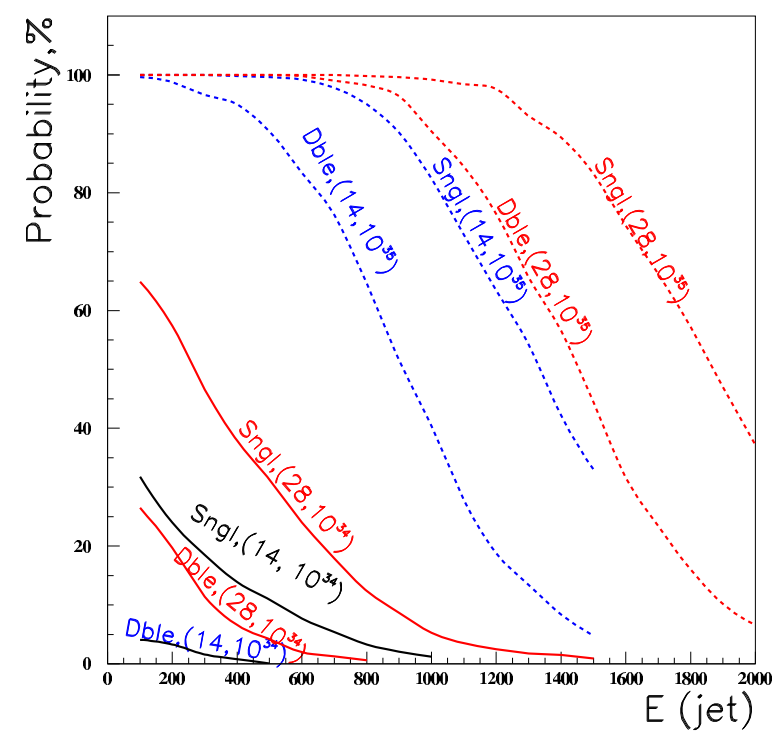

FIG. 8: Probability of single and double fake forward jet tagging from pile-up at $\mathcal{L}=10^{34} \mathrm{~cm}^{-2} \mathrm{~s}^{-1}$ (solid) and $\mathcal{L}=10^{35} \mathrm{~cm}^{-2} \mathrm{~s}^{-1}$ (dashed) for $p p$ collisions at $\sqrt{s}=$ $14 \mathrm{TeV}$ and $\sqrt{s}=28 \mathrm{TeV}$ as a function of the jet energy (in $\mathrm{GeV}$ ). The figure is taken from Ref. [3].

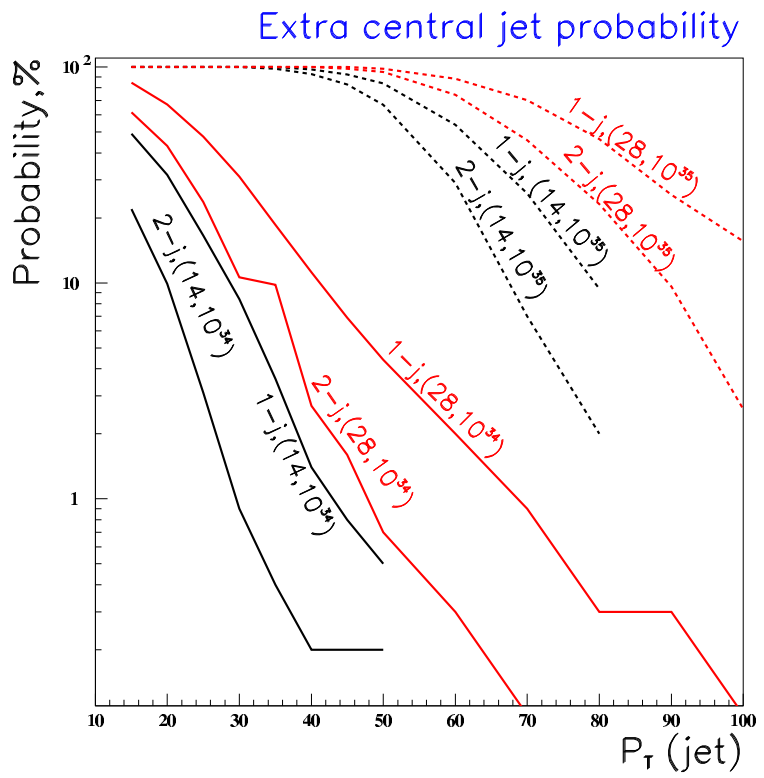

FIG. 9: Probability of observing one or two extra central jets from pile-up for $\mathcal{L}=10^{34} \mathrm{~cm}^{-2} \mathrm{~s}^{-1}$ (solid) and $\mathcal{L}=10^{35} \mathrm{~cm}^{-2} \mathrm{~s}^{-1}$ (dashed) at $\sqrt{s}=14 \mathrm{TeV}$ and $\sqrt{s}=28 \mathrm{TeV}$ as a function of jet transverse energy (in $\mathrm{GeV})$. The figure is taken from Ref. [3].

\section{Supersymmetry}

\section{SUGRA Models}

If supersymmetry is connected to the hierarchy problem, it is expected that sparticles will be sufficiently light that at least some of them will be observable at the LHC. As the sparticle masses rise, the fine tuning problem of the SM reappears. If supersymmetry is also the solution to the dark matter problem, the stable lightest supersymmetric particle (LSP) is the particle that pervades the universe. This constraint can be applied to the minimal SUGRA model in order to constrain the masses of the other sparticles. Recently, minimal SUGRA points have been proposed [40] which satisfy existing constraints, including the dark matter constraint, but do not impose any fine tuning limits. Most of the allowed parameter space corresponds to the case where the sparticle masses are less than about $1 \mathrm{TeV}$ and thus is accessible to the LHC. For an integrated luminosity of $100 \mathrm{fb}^{-1}$, squarks and gluinos with masses up to about $2 \mathrm{TeV}$ can be discovered at the LHC. For $1 \mathrm{ab}^{-1}$, the mass reach improves by $15-30 \%$, while doubling the energy of the LHC would also double the mass reach for squarks and gluinos to $\approx 4 \mathrm{TeV}[3]$. These limits are essentially model independent.

However, there are regions of parameter space where the annihilation rate for the LSP can be increased; in these regions the sparticle masses can be much larger. Two of these points in parameter space, $\mathrm{K}$ and L, are discussed in Ref. 41. Here, we concentrate on point $M$ which is characterized by very large sparticle masses: except for the $h^{0}$ and $\chi_{1}^{0}$, all sparticles have masses larger than $1.2 \mathrm{TeV}$ 40, 41]. We consider a luminosity upgraded LHC and the VLHC-I with $\sqrt{s}=40 \mathrm{TeV}$. For the purposes of this simulation, we assume that the detector performance at $\mathcal{L}=10^{35} \mathrm{~cm}^{-2} \mathrm{~s}^{-1}$ is the same as that of the ATLAS detector at the LHC design luminosity. Additional pile-up is taken into account by raising some of the cuts. Only the $\bar{t} t, W j$ and $Z j$ backgrounds are included in the study reported here. Events are selected with hadronic jets and missing $E_{T}$ and the following scalar quantity formed

$$
M_{\text {eff }}=\not_{T}+\sum_{\text {jets }} E_{T}(j e t)+\sum_{\text {leptons }} E_{T}(\text { lepton }),
$$

where the sum extends over all jets with $E_{T}>50 \mathrm{GeV}$ and $|\eta|<5$, and isolated leptons with $E_{T}>15 \mathrm{GeV}$ 

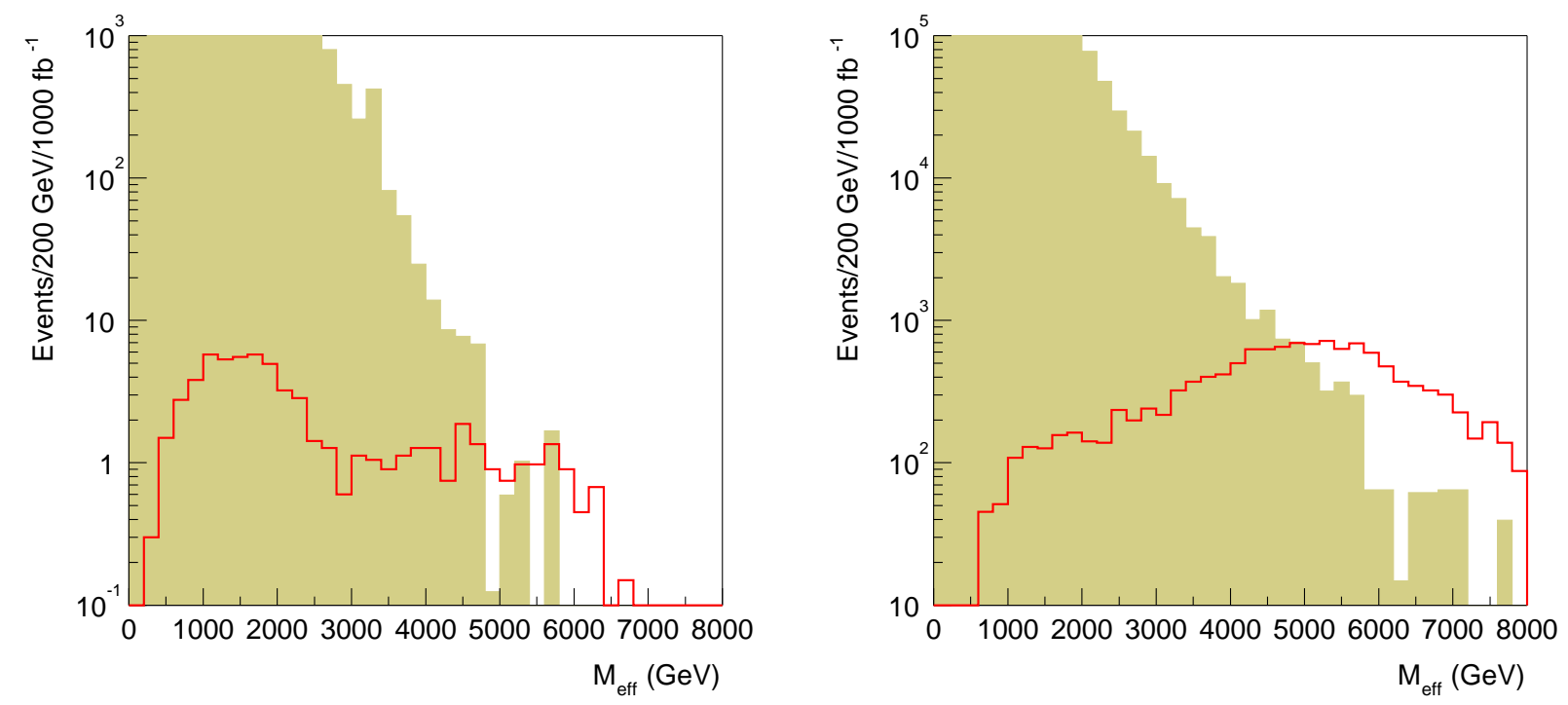

FIG. 10: $M_{\text {eff }}$ distribution for SUGRA point M at the SLHC (left) and the VLHC-I (right). The solid (shaded) histogram represents the signal (SM background).

and $|\eta|<2.5$. At least two jets with

$$
p_{T}(j)>0.1 M_{e f f}, \quad E_{T}>0.3 M_{e f f}, \quad \Delta \phi\left(j_{1}, E_{T}\right)<\pi-0.2, \quad \text { and } \quad \Delta \phi\left(j_{1}, j_{2}\right)<\frac{2 \pi}{3}
$$

are required.

Due to the large sparticle masses for point M, only 375 supersymmetric events are produced at the LHC even with an integrated luminosity of $1 \mathrm{ab}^{-1}$. At the VLHC-I, the cross section is about a factor 200 larger. The $M_{\text {eff }}$ distributions at the SLHC and VLHC-I are shown in Fig. 10. The SLHC signal is very marginal. For $M_{\text {eff }}>5 \mathrm{TeV}$, there only 6.4 signal events with 3.5 background events. The VLHC-I signal is clearly visible and could be further optimized.

\section{Inverted Mass Hierarchy Models}

Taking arbitrary weak scale soft supersymmetry breaking parameter choices generally leads to conflict with various low energy constraints associated with flavor changing neutral currents (FCNC). Three possibilities have emerged for building models consistent with low energy constraints:

1. universality of scalar lepton masses. The MSUGRA model adopts universality as an ad hoc assumption.

2. alignment of fermion and sfermion mass matrices, and

3. decoupling, which involves setting sparticle masses to such high values that loop effects from sparticles are suppressed relative to SM loops [42].

It is important to notice that "naturalness" arguments, which generally require sparticle masses $<1 \mathrm{TeV}$ most directly apply to third generation superpartners, owing to their large Yukawa couplings. In contrast, the FCNC constraints mainly apply to the scalar masses of the first two generations. This observation has motivated the construction of so-called inverted mass hierarchy (IMH) models, where the squarks and sleptons associated with the first two generations have multi-TeV masses, while third generation scalars have sub-TeV masses. Models where the IMH is already in place at the grand unification scale [43] typically have first and second generation scalar masses in the $5-20 \mathrm{TeV}$ range and rather heavy charginos, neutralinos and gluinos, making their discovery a considerable challenge at the LHC and a LC. The cross section for squark pair production (assuming four degenerate squark flavors) for $\sqrt{s}=200 \mathrm{TeV}$ as a function of the squark mass is shown in Fig. 11 (solid line). Assuming an integrated luminosity of $200 \mathrm{fb}^{-1}$, it should be possible to discover squarks in IMH models with masses up to about $20 \mathrm{TeV}$. 


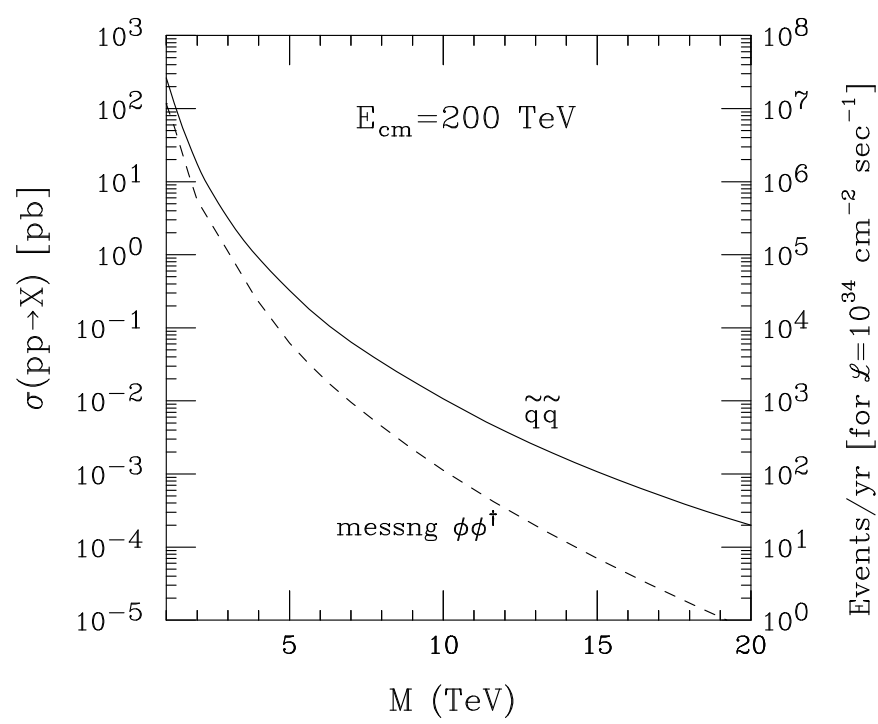

FIG. 11: The cross section for squark pair production (solid line) and pair production of vector-like messenger fields (dashed line) in $p p$ collisions for $\sqrt{s}=200 \mathrm{TeV}$. The calculation assumes four degenerate squark flavors, and two degenerate flavors for the $\Phi$ fields.

\section{Probing the Dynamics of Supersymmetry Breaking at the VLHC}

Any supersymmetric theory of physics beyond the SM must contain a mechanism for breaking supersymmetry and a method (messenger) for communicating this breaking to the superpartners of the SM particles. Hence, any discovery of supersymmetry immediately implies the existence of two, possibly distinct, new scales: the fundamental scale of supersymmetry breaking given by the dimension-two vacuum expectation value $F$, and the messenger scale $M$. A typical superpartner mass $\widetilde{m}$ is related to $M$ and $F$ by [44]

$$
\widetilde{m} \sim \eta \frac{F}{M}
$$

where $\eta$ is a possible suppression factor originating from dimensionless couplings. In SUGRA models, $M=$ $M_{P l} \approx 10^{19} \mathrm{GeV}$ and $\sqrt{F} \sim 10^{10} \mathrm{GeV}$.

If supersymmetry breaking is communicated by gauge interactions (GMSB models), $M$ is replaced by the mass of heavy messenger fields, $\Phi$, and the parameter $\eta$ contains a factor $\alpha / 4 \pi$. The messenger fields form a vector-like representation of the gauge group. If $\sqrt{F} \sim M$ and $\widetilde{m} \lesssim 1 \mathrm{TeV}$, it is possible that the mass of the $\Phi$ fields is in the range of $10-100 \mathrm{TeV}$. It may thus be possible to directly produce the messenger fields at a VLHC. The cross section for pair production of colored spin- 0 messenger fields at $\sqrt{s}=200 \mathrm{TeV}$ is given by the dashed line in Fig. 11. For the VLHC-II design luminosity of $\mathcal{L}=2 \cdot 10^{34} \mathrm{~cm}^{-2} \mathrm{~s}^{-1}$ at this energy [4], it is clear that it will be difficult to discover messenger fields which are heavier than about $12 \mathrm{TeV}$. An additional factor of $\sim 5$ in luminosity would increase the maximum $\Phi$ mass which can be accessed to $\sim 18 \mathrm{TeV}$.

If the messenger fields do not directly couple to the SM fields, the "messenger number" is conserved, and the lightest messenger particle (LMP), $\Phi_{0}$, is stable. In this scenario, the LMP has to be lighter than a few TeV in order not to overclose the universe in the standard inflationary cosmology 45. The heavier messenger fields decay into the LMP and a SM gauge field, $V=W, Z, \gamma, g$,

$$
\Phi \rightarrow \Phi_{0}+V \rightarrow \not_{T}+V
$$

$\Phi$ pair production thus is signaled by pair production of SM gauge fields accompanied by a large amount of missing transverse energy from the LMP's escaping the detector.

If the messenger fields couple to SM multiplets [46], the LMP can decay, e.g. via

$$
\Phi_{0} \rightarrow e \mu, \tilde{q}_{i} \tilde{q}_{j}, h \tilde{q} .
$$

$\Phi_{0} \bar{\Phi}_{0}$ production thus can result in $e^{+} e^{-} \mu^{+} \mu^{-}$events with a bump in the invariant mass of the $e \mu$ systems. The decays into two squarks, or a Higgs boson and a squark, together with the subsequent $h$ or $\tilde{q}$ cascade decays, can lead to events with very high particle multiplicities in the final state. 
The scales $\sqrt{F}$ and $M$ can be determined experimentally. In GMSB models, the gravitino, $\tilde{G}$, is the lightest supersymmetric particle and its mass is very small. The phenomenology of the superpartners of the SM gauge and fermion fields is then determined by the nature of the next lightest supersymmetric particle (NLSP), and by its lifetime and decay into a $\tilde{G}[21]$. The lifetime of the NLSP,

$$
c \tau_{N L S P}=100 \mu m\left[\frac{100 \mathrm{GeV}}{m_{N L S P}}\right]^{5}\left[\frac{\sqrt{F}}{100 \mathrm{TeV}}\right]^{4},
$$

measures the overall supersymmetry breaking scale and thus is a crucial parameter in these models. From the sparticle mass spectrum it is then possible (see Eq. (7)) to determine the messenger scale $M$. At the LHC, it should be possible to determine $\sqrt{F}$ with a precision of about $10 \%$, and $M$ with a precision of $\sim 30 \%$ [4]. If experiments at the LHC discover supersymmetry and determine that $M<20 \mathrm{TeV}$, the messenger fields $\Phi$ could be directly produced at the VLHC. Such a scenario would provide a compelling physics case for a hadron collider operating in the $100 \mathrm{TeV}$ range.

\section{Strong Electroweak Symmetry Breaking}

While the existing precision electroweak measurements prefer a light Higgs boson [19, 48], the possibility of electroweak symmetry breaking by new strong dynamics at the TeV scale cannot be excluded.

\section{Strongly Interacting Weak Bosons}

The couplings of longitudinally polarized gauge bosons to each other are fixed at low energy by the nature of the spontaneously broken electroweak symmetry and are independent of the details of the mechanism which breaks the $S U(2)_{L} \times U(1)_{Y}$ symmetry. Weak boson scattering amplitudes calculated from these couplings violate $S$-matrix unitarity at center of mass energies of $\sim 1.5 \mathrm{TeV}$. To cure this problem, new physics must enter. In the SM and supersymmetric theories, the cure arises from one or several weakly coupled Higgs bosons. If no Higgs-like particles exist, new strong dynamics must enter in the weak boson scattering amplitudes at high energies.

Various models exist which can be used as benchmarks for new strong interactions. The basic signal for all these models is an excess of events over that predicted by the SM for gauge boson pairs at large invariant masses produced in vector boson scattering processes. In some models such as technicolor [49, 50] or topcolor-assisted technicolor [51, resonances appear, in others the new strong dynamics is signaled by a smooth enhancement over the SM cross section. This more difficult case has been studied in detail by the ATLAS collaboration for $W^{ \pm} W^{ \pm}$production via WBF at an upgraded LHC [3] for the K-matrix unitarization model [52]. In this model, $S$-matrix unitarity is achieved by replacing the partial wave amplitudes, $a_{\ell}^{I}$, by

$$
t_{\ell}^{I}=\frac{a_{\ell}^{I}}{1-i a_{\ell}^{I}}
$$

where $I$ is the isospin, and $\ell$ is the angular momentum quantum number.

Events were selected that have $\not_{T}>40 \mathrm{GeV}$ and two same sign leptons with $p_{T}(\ell)>40 \mathrm{GeV}\left(p_{T}(\ell)>50 \mathrm{GeV}\right.$ for $\sqrt{s}=28 \mathrm{TeV}$ ) and $|\eta(\ell)|<1.75$. Backgrounds from $W Z$ and $Z Z$ production were rejected by requiring that no third lepton be present which, in combination with one of the other leptons, is consistent with the decay of a $Z\left(\left|M_{Z}-m\left(\ell^{+} \ell^{-}\right)\right|<15 \mathrm{GeV}\right)$. In addition, the two leptons are required to have $m(\ell \ell)>100 \mathrm{GeV}$, $\Delta \phi(\ell \ell)<-0.5(\Delta \phi(\ell \ell)<-0.8$ for $\sqrt{s}=28 \mathrm{TeV})$, and, for $\sqrt{s}=28 \mathrm{TeV}, \Delta p_{T}(\ell \ell)>100 \mathrm{GeV}$. A jet veto requiring no jets with $p_{T}(j)>50 \mathrm{GeV}$ and $|\eta(j)|<2$ is effective against $W t \bar{t}$ production. Requiring two forward jets with $|\eta(j)|>2$ reduces the background from di-boson production. Jet tagging (vetoing) in the forward (central) region is essential to extract the signal. The resulting signal and background distributions as a function of the invariant mass formed from the two leptons and the missing transverse momentum for $\sqrt{s}=28 \mathrm{TeV}$ are shown in Fig. 12. Both signal and background distributions are seen to have similar shapes, which will lead to rather large systematic uncertainties.

The resulting statistical significances for $300 \mathrm{fb}^{-1}$ as a function of $\sqrt{s}$ are shown in Fig. 13. At the LHC, the statistical significance of the K-matrix unitarization model is marginal $(S / \sqrt{S+B}=2.9)$. Increasing the luminosity of the LHC by a factor 10 above the design luminosity does not improve $S / \sqrt{S+B}$. As discussed in Sec. II B 6, the jet veto and jet tagging thresholds have to be increased substantially in this case, due to the pile-up from the increased number of interactions per crossing. This effectively reduces the number of useful 


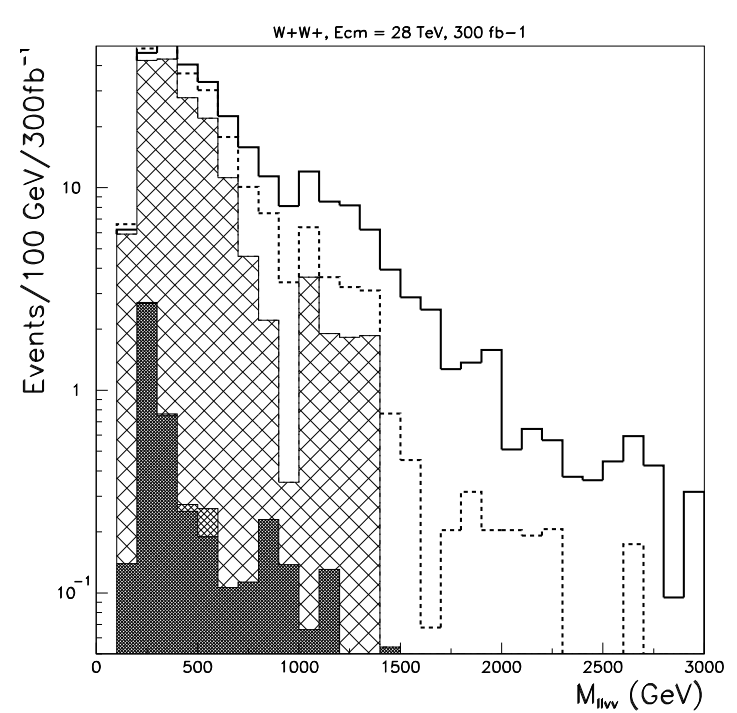

FIG. 12: Signals from strong $W^{ \pm} W^{ \pm}$scattering at $\sqrt{s}=28 \mathrm{TeV}$ for an integrated luminosity of $300 \mathrm{fb}^{-1}$. Event rates are shown as a function of the invariant mass of the $\ell \ell \mathbb{E}_{T}$ system. The backgrounds are shown as histograms, from inside to outside (or darker to lighter): $W Z$ continuum, $W$ pair production from gluon exchange, and $W$ pair production from photon and $Z$ exchange. The solid line shows the signal from K-matrix unitarization. For comparison, the dashed line displays the signal for a SM Higgs boson with $M_{H}=1 \mathrm{TeV}$ (from Ref. [3]).

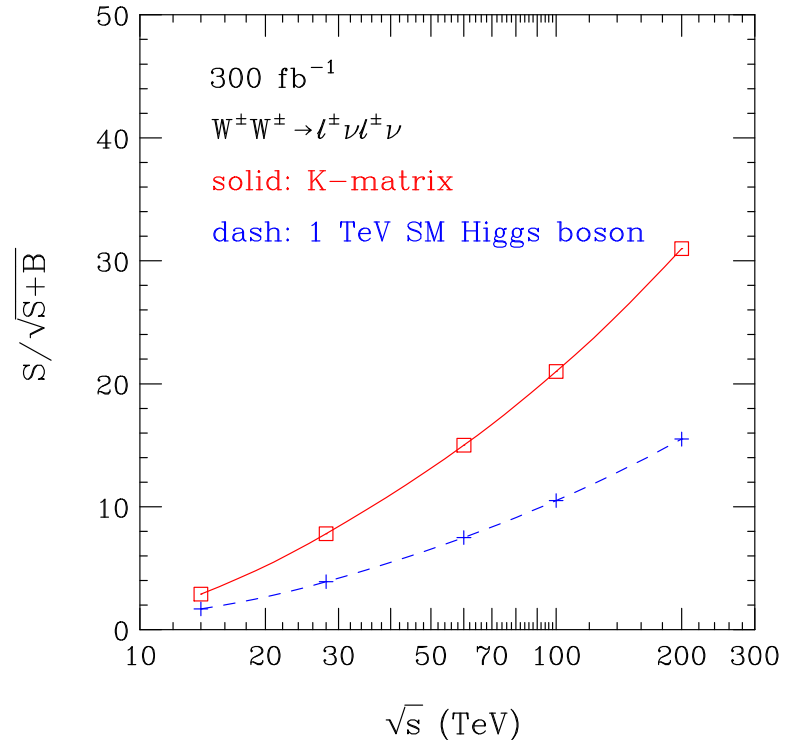

FIG. 13: Significance of the signal for the K-matrix unitarization model (solid line) in $W^{ \pm} W^{ \pm} \rightarrow \ell^{ \pm} \nu \ell^{ \pm} \nu$ as a function of the center of mass energy, $\sqrt{s}$, for an integrated luminosity of $300 \mathrm{fb}^{-1}$. For comparison the result for a SM Higgs boson with $M_{H}=1 \mathrm{TeV}$ (dashed line) in this channel is also shown.

events to approximately that present at the LHC design luminosity [3]. At the VLHC-I, on the other hand, the signal stands out clearly $(S / \sqrt{S+B} \approx 11)$.

\section{Technicolor and Topcolor}

Many models of strong electroweak symmetry breaking predict the existence of new heavy fermions. Here we briefly discuss two examples.

In technicolor models [49, 50], the existence of new quarks bound by novel strong interactions is predicted. A condensate of these so-called techniquarks, $Q_{T C}$, breaks the $S U(2)_{L} \times U(1)_{Y}$ symmetry. Techniquarks can be pair produced in hadronic collisions via ordinary strong interactions. Assuming that the techniquarks are in the fundamental representation of the group $S U(4)_{T C}$ which is supposed to dynamically break the electroweak symmetry, we show the cross section for $Q_{T C}$ pair production as a function of the $Q_{T C}$ mass for $\sqrt{s}=200 \mathrm{TeV}$ in Fig. 14 (solid line). It should be possible to detect techniquarks with masses up to about $15 \mathrm{TeV}$ at a VLHC-II with an integrated luminosity of $100 \mathrm{fb}^{-1}$.

The top-seesaw model [53, 54], a variant of the original [51] topcolor model, predicts the existence of partners of the top quark, $\chi_{R}$ and $\chi_{L}$, which transform as singlets under $S U(2)_{L}$. These fermions are responsible for the dynamical breaking of the electroweak symmetry. Current precision data constrain their masses to the range $3 \mathrm{TeV} \lesssim M_{\chi} \lesssim 10 \mathrm{TeV}$ 54]. The cross section for $\chi$ pair production in $p p$ collisions at $\sqrt{s}=200 \mathrm{TeV}$ is given by the dashed line in Fig. 14, assuming that the $\chi_{L}$ and $\chi_{R}$ are degenerate in mass. For $M_{\chi}=10 \mathrm{TeV}$ and an integrated luminosity of $100 \mathrm{fb}^{-1}$, approximately 100 events are expected. If the electroweak symmetry is dynamically broken via the mechanism suggested by top-seesaw models, the VLHC-II will be able to directly produce the particles which trigger the breaking of $S U(2)_{L} \times U(1)_{Y}$. 


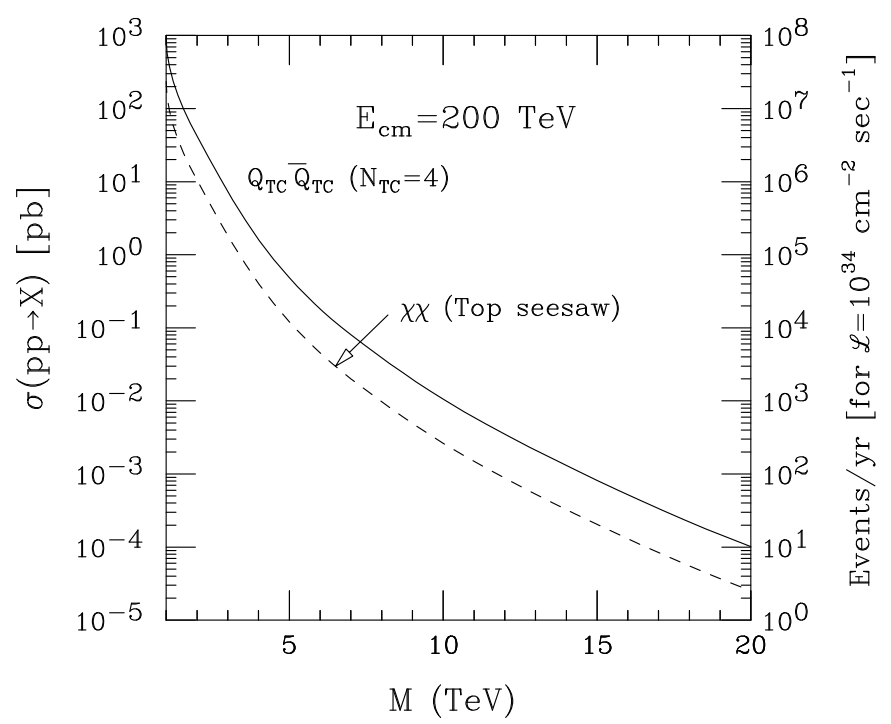

FIG. 14: The cross section for $Q_{T C}$ pair production (solid line) and pair production of $S U(2)_{L}$ singlet top quark partners $\chi_{L}$ and $\chi_{R}$ in topcolor models (dashed line) in $p p$ collisions at $\sqrt{s}=200 \mathrm{TeV}$. The calculation assumes one degenerate isodoublet of techniquarks, and $\chi_{L}$ and $\chi_{R}$ states which are degenerate in mass. QCD corrections are simulated by taking into account a $k$-factor of $k=2$. The right vertical scale shows the number of events per year expected for a luminosity of $\mathcal{L}=10^{34} \mathrm{~cm}^{-2} \mathrm{~s}^{-1}$.

\section{E. Extra Gauge Bosons}

The existence of heavy neutral $\left(Z^{\prime}\right)$ and/or charged $\left(W^{\prime}\right)$ vector bosons is a feature of many extensions of the SM. They arise in extended gauge theories including grand unified theories, superstring theories, left-right symmetric models, and other models such as the BESS model [55] and models of composite gauge bosons (for a review see Ref. [56]). The mass reach depends on the couplings of the $W^{\prime}$ and $Z^{\prime}$ bosons to quark and leptons.

The search limits for $Z^{\prime}$ bosons at the Tevatron, LHC, SLHC and VLHC for various integrated luminosities are shown in Fig. 15 [57. These bounds are based on requiring $10 Z^{\prime} \rightarrow \ell^{+} \ell^{-}(\ell=e, \mu)$ events and are in good agreement with limits obtained by CDF [60] and $\mathrm{D} \varnothing[61$. The discovery limits in general improve by about $20 \%$ if the integrated luminosity is increased from $100 \mathrm{fb}^{-1}$ to $1 \mathrm{ab}^{-1}$. Note that the search reaches of a SLHC with $\sqrt{s}=28 \mathrm{TeV}$ and $1 \mathrm{ab}^{-1}$ and a VLHC-I with $\sqrt{s}=40 \mathrm{TeV}$ and $100 \mathrm{fb}^{-1}$ are similar. For $\sqrt{s}=100 \mathrm{TeV}$ $(\sqrt{s}=200 \mathrm{TeV})$ and $100 \mathrm{fb}^{-1}, Z^{\prime}$ bosons with masses up to $20-25 \mathrm{TeV}(30-40 \mathrm{TeV})$ can be discovered. Qualitatively similar results are obtained for $W^{\prime}$ bosons. At an $e^{+} e^{-}$or muon collider, direct searches for a $Z^{\prime}$ boson are limited to the region $M_{Z^{\prime}}<\sqrt{s}$.

\section{F. Compositeness}

Composite models of quarks and leptons attempt to overcome the shortcomings of the SM by assuming that they are bound states of more fundamental fermions, and perhaps bosons, bound together by a new interaction which is characterized by an energy scale $\Lambda$. At energies much smaller than $\Lambda$, the substructure of quarks and leptons is signalled by the appearance of four fermion contact interactions which arise from the exchange of bound states of the subconstituents [62]. For energies similar to or larger than $\Lambda$, one expects that excited states of the known quarks and leptons are produced [63, 64].

\section{Contact Interactions}

The lowest order contact terms are dimension 6 four-fermion interactions which can affect jet and Drell-Yan production at hadron colliders. Compared with the SM terms, they are suppressed by a factor $1 / \Lambda^{2}$. The signature for four quark contact interactions, for example, would be an excess of events at large transverse energy, $E_{T}$. Another signal for quark - quark contact interactions, which, in contrast to the $E_{T}$ distribution, is 


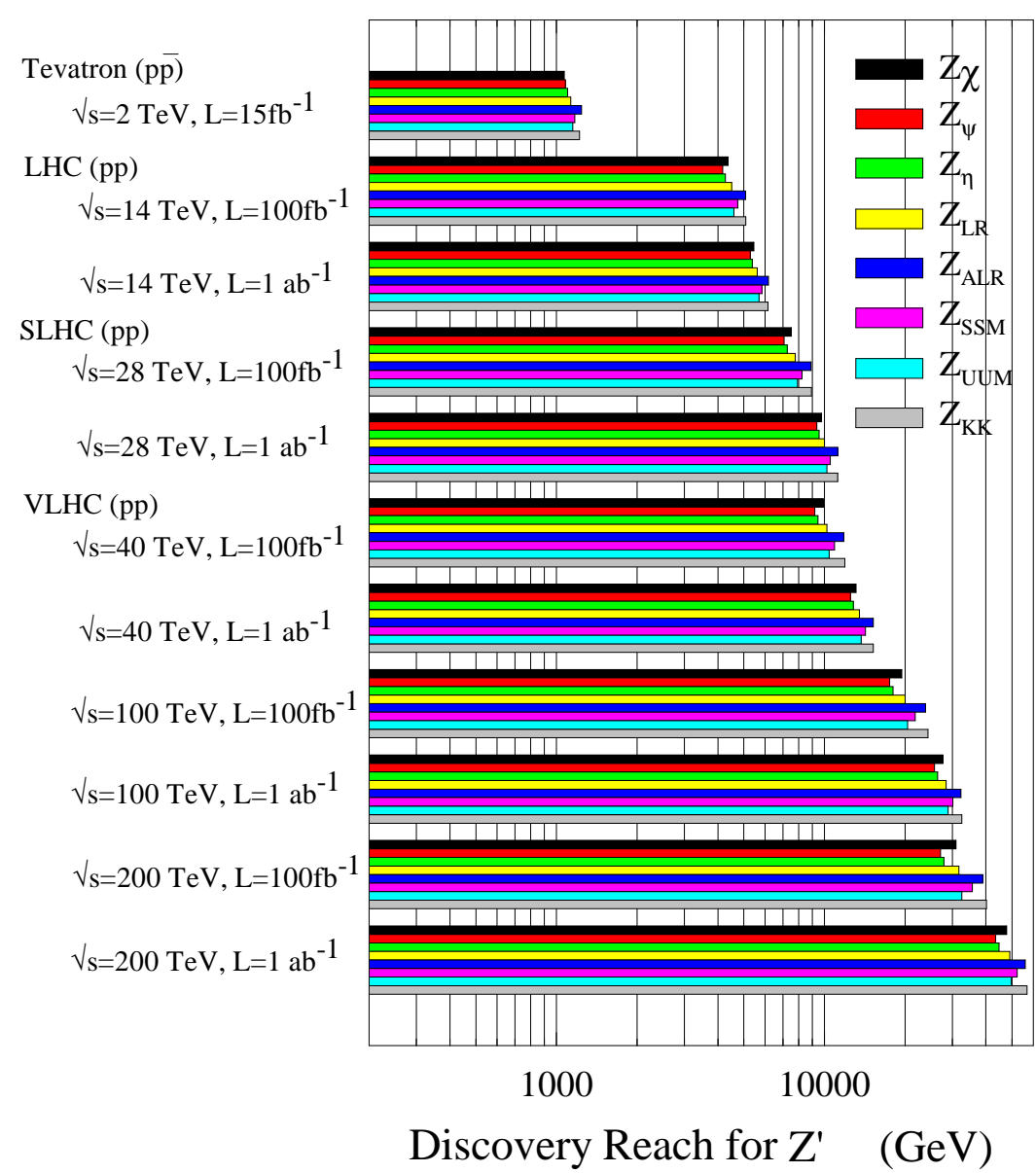

FIG. 15: Discovery limits for extra neutral gauge bosons $\left(Z^{\prime}\right)$ for a variety of models. $Z_{\chi}, Z_{\psi}$ and $Z_{\eta}$ refer to the $Z^{\prime}$ bosons predicted by grand unified models of rank 5 , such as $S O(10)$ or $E(6)$ [56]. $Z_{L R}$ is the extra gauge boson of left-right symmetric models embedded in $S O(10)$ [56]. The alternative left-right symmetric model of Ref. [58] predicts an extra neutral gauge boson, $Z_{A L R}$. The $Z_{S S M}$ case corresponds to a $Z^{\prime}$ with SM like couplings. The $Z_{U U M}$ is the extra gauge boson of the un-unified model of Ref. [59]. Finally, the $Z_{K K}$ refers to the lowest Kaluza-Klein excitation of the SM $Z$ boson which appears in models with $\mathrm{TeV}$-scale extra dimensions (see Sec. II G 2). The discovery limits are based on 10 events in the $e^{+} e^{-}$and $\mu^{+} \mu^{-}$channels (from Ref. [57]).

not sensitive to theoretical or jet energy uncertainties, is the dijet angular distribution, $d \sigma / d \chi$, where

$$
\chi=\frac{1+|\cos \theta|}{1-|\cos \theta|}
$$

with $\theta$ being the angle between a jet and the beam in the center of mass of the dijet system. If contact terms are present, the dijet angular distribution is more isotropic than that predicted by QCD. Figure 16 shows the deviation of the angular distribution from the SM prediction at an upgraded LHC. The maximum compositeness scales which can be probed using $d \sigma / d \chi$ are shown in Table II. No detailed study of what values of $\Lambda$ could be probed at a VLHC has been carried out so far. Extrapolating the limits listed in Table II to $\sqrt{s}=200 \mathrm{TeV}$, one finds that one should be able to probe values of $\Lambda \approx 100 \mathrm{TeV}$ at the VLHC-II with an integrated luminosity of $300 \mathrm{fb}^{-1}$.

\section{Excited Quarks}

Conclusive evidence for a new layer of substructure would be provided by the direct observation of excited states of the known quarks and leptons. In the following we shall concentrate on excited quarks with spin $1 / 2$ 


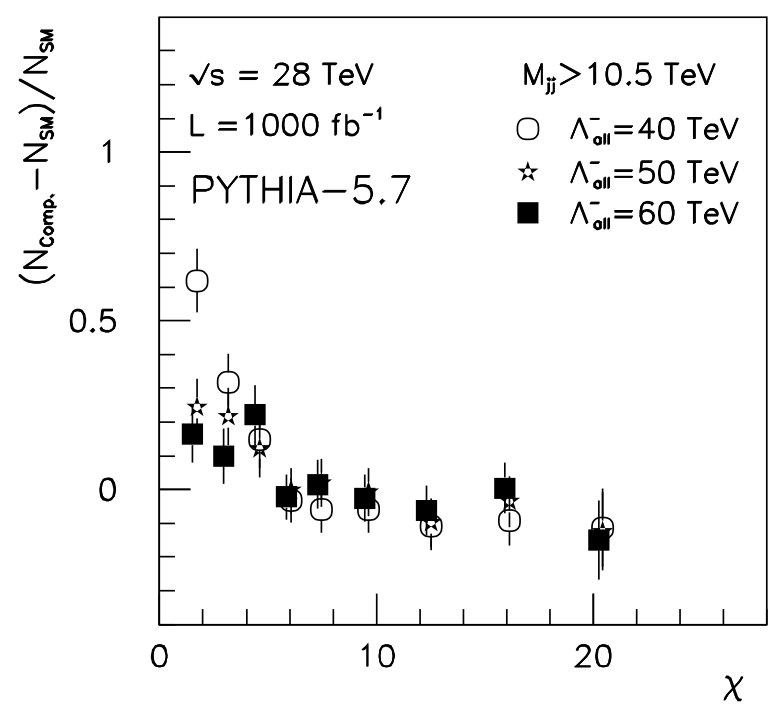

FIG. 16: Deviation from the SM prediction for the angular distribution of dijet pairs at $\sqrt{s}=28$ TeV for various values of $\Lambda$. Dijet pairs are required to have invariant mass $M_{j j}>10.5 \mathrm{TeV}$ (from Ref. [3]). Constructive interference between $\mathrm{SM}$ and contact terms is assumed in the matrix elements.

TABLE II: The 95\% confidence level limits that can be obtained for $\Lambda$ using the dijet angular distribution (from Ref. 33).

\begin{tabular}{|c|c|c|c|c|}
\hline $\begin{array}{c}\text { energy } \\
\int \mathcal{L} d t\end{array}$ & $\begin{array}{c}\sqrt{s}=14 \mathrm{TeV} \\
300 \mathrm{fb}^{-1}\end{array}$ & $\begin{array}{c}\sqrt{s}=14 \mathrm{TeV} \\
3000 \mathrm{fb}^{-1}\end{array}$ & $\begin{array}{c}\sqrt{s}=28 \mathrm{TeV} \\
300 \mathrm{fb}^{-1}\end{array}$ & $\begin{array}{c}\sqrt{s}=28 \mathrm{TeV} \\
3000 \mathrm{fb}^{-1}\end{array}$ \\
\hline$\Lambda(\mathrm{TeV})$ & 40 & 60 & 60 & 85 \\
\hline
\end{tabular}

and weak isospin 1/2. Excited up- and down-quarks should be almost degenerate in mass if the dynamics which binds the quark constituents respects isospin. The coupling between excited spin $1 / 2$ quarks, ordinary quarks and gauge bosons is uniquely fixed to be of magnetic moment type by gauge invariance [63],

$$
\mathcal{L}_{e f f}=\frac{1}{2 m^{*}} \bar{q}^{*} \sigma^{\mu \nu}\left[g_{s} f_{s} \frac{\lambda^{a}}{2} F_{\mu \nu}^{a}+g f \frac{\vec{\tau}}{2} \vec{W}_{\mu \nu}+g^{\prime} f^{\prime} \frac{Y}{2} B_{\mu \nu}\right] q_{L}+\text { h.c. . }
$$

Here, $q^{*}$ and $q_{L}$ denote the isospin doublets of excited and lefthanded ordinary quarks, $V_{\mu \nu}, V=F^{a}, \vec{W}, B$, is the field strength tensor for the gluon, the $S U(2)$ and the $U(1)$ gauge fields, and $Y=1 / 3$ is the weak hypercharge. Finally, $g_{s}, g$ and $g^{\prime}$ are the gauge couplings and $f_{s}, f$ and $f^{\prime}$ are free parameters determined by the composite dynamics. Naively one would expect that they are all of $\mathcal{O}(1)$. To set the scale in $\mathcal{L}_{\text {eff } f}$ we choose the $q^{*}$-mass $m^{*}$.

Excited quarks decay into quarks and a gluon, photon or $W / Z$ boson, or, via contact interactions into $\bar{q} q q^{\prime}$ final states 64. Subsequently, only decays via gauge interactions are considered. Excited quarks are then expected to decay predominantly via strong interactions; $q^{*} \rightarrow q \gamma, q^{*} \rightarrow q^{\prime} W$ and $q^{*} \rightarrow q Z$ will typically appear at the few per cent level.

In hadronic collisions, excited quarks can be produced singly via quark gluon fusion. The subsequent $q^{*} \rightarrow q g$ decay leads to a peak in the two jet invariant mass distribution located at $m(j j)=m^{*}$. At the LHC, with $100 \mathrm{fb}^{-1}$, excited quarks with masses up to $m^{*}=6.6 \mathrm{TeV}$ can be discovered [3, 65]. For $\sqrt{s}=28 \mathrm{TeV}$, the reach can be increased to $m^{*} \approx 10 \mathrm{TeV}[3]$. At the VLHC, much higher masses can be probed. The dijet invariant mass distribution for $p p$ collisions at $\sqrt{s}=200 \mathrm{TeV}$, assuming $f_{s}=f=f^{\prime}=1$ and $m_{u^{*}}=m_{d^{*}}$, is shown in Fig. 17. PYTHIA 6.158 66 has been used for the simulation and both jets are required to have $p_{T}(j)>10 \mathrm{TeV}$ and $|\eta(j)|<2$. Detector resolution effects are simulated by taking a constant term of $C=0.05$ in the jet energy resolution into account. The sampling term in the jet energy resolution will have a very small effect at the energies considered here. The significance, $S / \sqrt{B}$ as a function of the excited quark mass, $m^{*}$, is shown in Fig. 18. Assuming a minimum significance of $S / \sqrt{B}=5$ to claim discovery, one concludes that, depending on the detector resolution, excited quarks with a mass up to $70-75 \mathrm{TeV}$ can be found at a $p p$ collider with $\sqrt{s}=200 \mathrm{TeV}$ and an integrated luminosity of $100 \mathrm{fb}^{-1}$. Similar results have been obtained in Ref. [67]. 


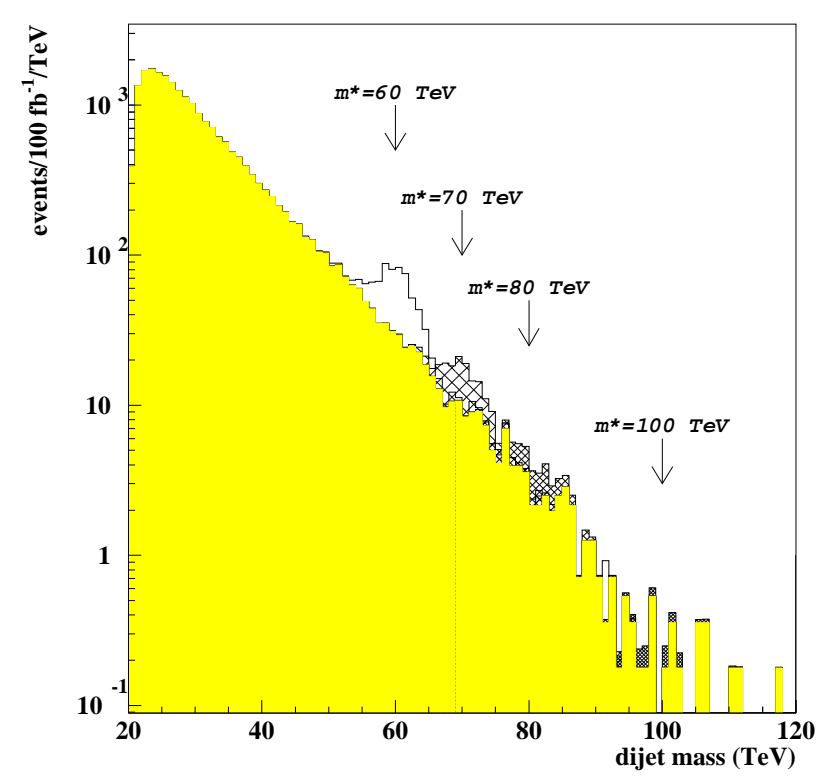

FIG. 17: Dijet invariant mass spectrum for $p p$ collisions at $\sqrt{s}=200 \mathrm{TeV}$, assuming an integrated luminosity of $100 \mathrm{fb}^{-1}$. Four excited quark resonances are shown above the QCD dijet background (shaded). To simulate detector response, a constant term $C=0.05$ in the jet energy resolution is assumed.

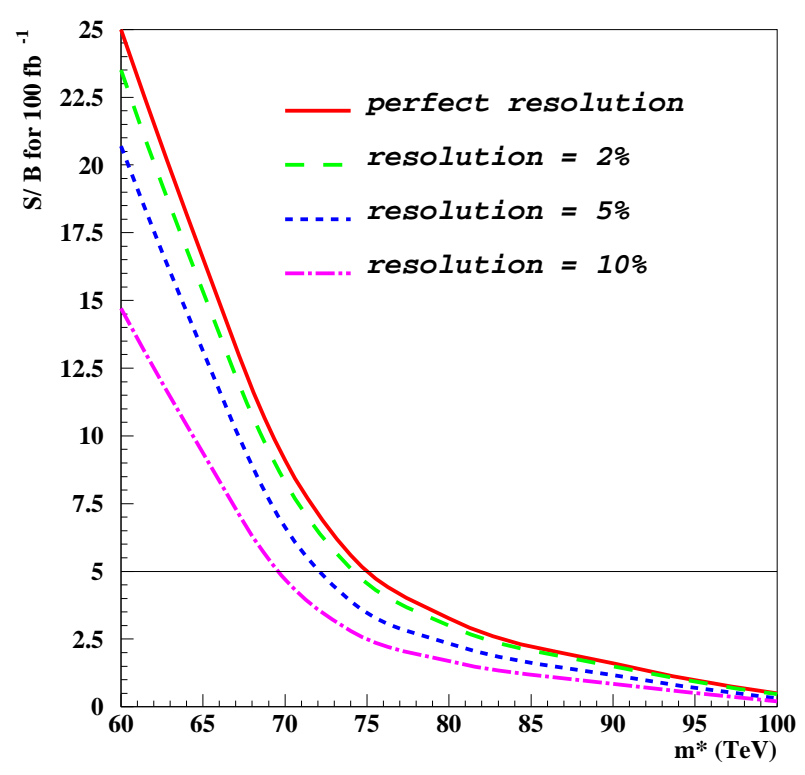

FIG. 18: Significance $S / \sqrt{B}$ as a function of $m^{*}$ for $p p$ collisions at $\sqrt{s}=200 \mathrm{TeV}, \int \mathcal{L} d t=100 \mathrm{fb}^{-1}$ and four different values of the jet energy resolution, $C=0$, $C=0.02, C=0.05$ and $C=0.1$.

The $q^{*}$ search in the dijet channel is limited mainly by the QCD background. In the $q^{*} \rightarrow q \gamma, q^{*} \rightarrow W q^{\prime}$ and $q^{*} \rightarrow Z q$ channels the signal is reduced by the relatively smaller branching fractions of these channels. However, the backgrounds are reduced as well. As a result, the $q^{*}$ mass reach in these channels is similar to that in the dijet channel. This has been verified by explicit studies for the LHC [68] and SLHC [3].

Should the (upgraded) LHC discover contact interactions in two jet production, the VLHC will be able to directly probe the scale of new physics.

\section{G. Extra Dimensions}

There is much recent theoretical interest in models of particle physics that have extra spatial dimensions in addition to the $3+1$ dimensions of normal space time. In these models, new physics can appear at a mass scale of $\mathcal{O}(1 \mathrm{TeV})$ and may be accessible at future hadron colliders. The models considered so far can be grouped in three classes which lead to very different phenomenologies and collider signatures.

1. The large extra dimension (ADD) scenario [69] which predicts the emission and exchange of Kaluza-Klein $(\mathrm{KK})$ towers of gravitons that are finely spaced in mass.

2. Models with TeV-scale extra dimensions ( $\mathrm{TeV}$ ) [70] which predict the existence of KK excitations of the SM gauge (and possibly other) fields at the $\mathrm{TeV}$ scale.

3. Models with warped extra dimensions such as the model of Ref. [71] (RS) which predict graviton resonances with both weak scale masses and couplings to matter.

In the following we briefly discuss these three classes as well as the production of black holes at hadron colliders.

\section{Large Extra Dimensions}

In models with large extra dimensions, massive graviton states can be produced in association with jets or photons. Since these graviton states have gravitational strength couplings, they escape the detector and thus 


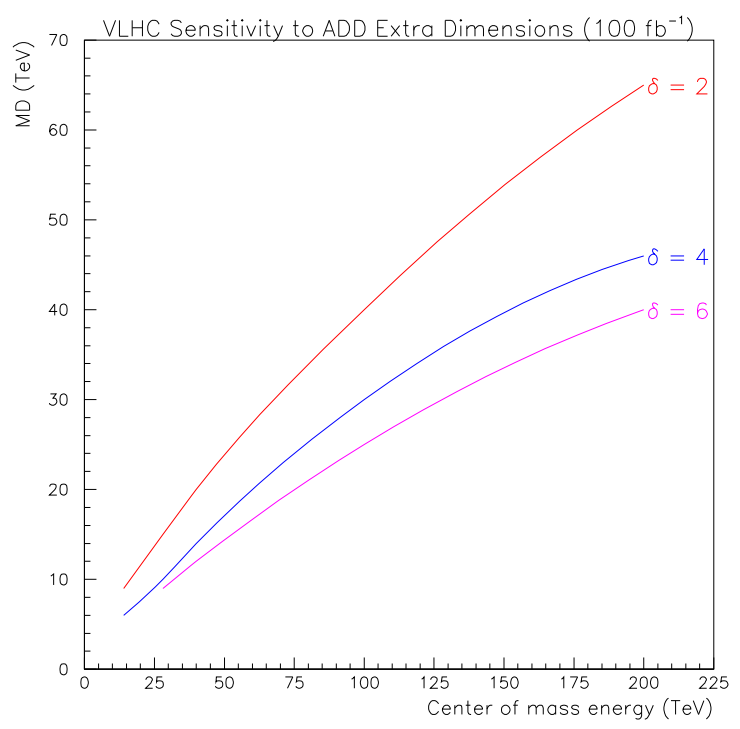

FIG. 19: $5 \sigma$ discovery limits that can be achieved for $M_{D}$ for an integrated luminosity of $100 \mathrm{fb}^{-1}$ as a function of $\sqrt{s}$. Results are shown for 2, 4 and 6 additional dimensions.

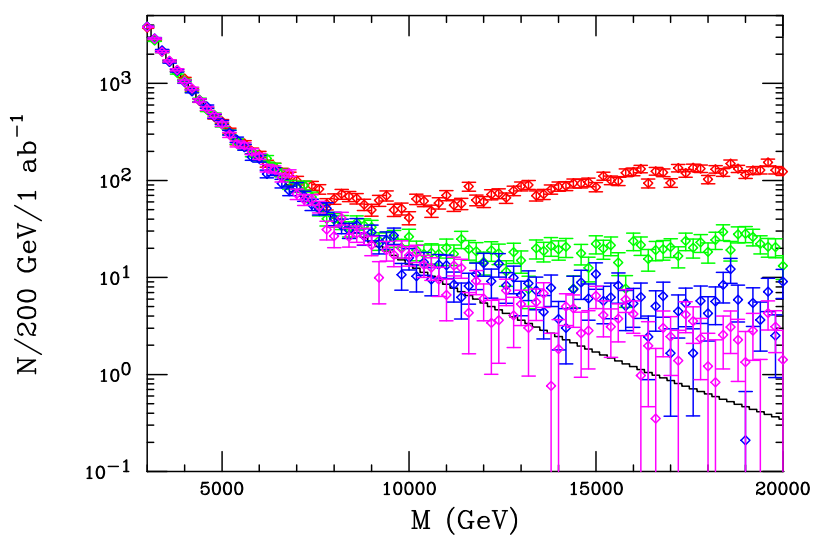

FIG. 20: Event rate per $200 \mathrm{GeV}$ mass bin for the Drell-Yan process, $p p \rightarrow \ell^{+} \ell^{-}$, as a function of the dilepton invariant mass for $\sqrt{s}=200 \mathrm{TeV}$ and $\int \mathcal{L} d t=$ $1 \mathrm{ab}^{-1}$. Both leptons are required to have rapidity $|\eta(\ell)|<2.5$. The solid histogram is the SM prediction whereas the 'data' points represent the predictions of the ADD model. The red, green, blue and magenta points (reading from top to bottom on the plot) correspond to $M_{s}=20,25,30$ and $35 \mathrm{TeV}$ respectively (from Ref. [76]).

give rise to missing transverse energy. The properties of the KK graviton tower are parameterized in terms of the number of additional dimensions, $\delta$, and the fundamental scale $M_{D}$. Since the $\not_{T}+$ jets final state is more sensitive it will be considered here. The background is dominated by $Z(\rightarrow \bar{\nu} \nu)+$ jets production. The maximum scale $M_{D}$ which can be accessed for $\delta=2, \delta=4$ and $\delta=6$ and an integrated luminosity of $100 \mathrm{fb}^{-1}$ is shown in Fig. 19 as a function of $\sqrt{s}$. At $\sqrt{s}=200 \mathrm{TeV}$, values of $M_{D}$ up to $65 \mathrm{TeV}(38 \mathrm{TeV})$ can be probed for $\delta=2(\delta=6)$. This is a factor $7-8$ higher than the scale which can be probed at the LHC with the same integrated luminosity [3, 72].

One can also search for indirect effects of the KK graviton towers appearing in ADD models, for example in Drell-Yan production [73]. In the SM, the Drell-Yan reaction is a result of photons and $Z$ bosons mediating the process $\bar{q} q \rightarrow \ell^{+} \ell^{-}(\ell=e, \mu)$. In the ADD model, graviton towers can also be exchanged, and an additional sub-process, $g g \rightarrow \ell^{+} \ell^{-}$, mediated solely by gravitons contributes. The effect of the graviton towers can be described through a set of dimension 8 operators in the limit that the parton center of mass energy is much larger than the cut-off scale, $M_{s}$, which is of order $M_{D}$. Current experimental data from LEP and the Tevatron [74] require that $M_{s} \geq 1 \mathrm{TeV}$, and values of $M_{s}$ as large as a few tens of $\mathrm{TeV}$ may be conceivable in this framework. The distortion of the differential cross section of the Drell-Yan process at large values of $m(\ell \ell)$ through these dimension 8 operators can probe such high mass scales in a manner similar to searches for contact interactions in composite models. The shape of the invariant mass distribution will tell us that the underlying physics arises from dimension 8 operators, while the angular distribution of the leptons at large di-lepton invariant masses would conform to the shape expected from the exchange of a spin-2 object, confirming the gravitational origin of the effect. At the LHC, values of $M_{s}$ up to $7.9 \mathrm{TeV}(7.0 \mathrm{TeV})$ can be probed in Drell-Yan production for 2 (4) extra dimensions with $100 \mathrm{fb}^{-1} 75$. The di-lepton invariant mass distribution for a VLHC operating at $\sqrt{s}=200 \mathrm{TeV}$ in the SM and for various values of $M_{s}$ is shown in Fig. 20. It is clear that values of $M_{s}$ up to about $35 \mathrm{TeV}$ can be probed at a VLHC. Similar mass scales can be probed at CLIC [77].

\section{TeV-scale Extra Dimensions}

In the simplest versions of $\mathrm{TeV}$-scale theories with extra dimensions, only the $\mathrm{SM}$ gauge fields are in the bulk whereas fermions remain at the orbifold fixed points. Higgs fields may lie at the fixed points or propagate in the bulk. In this simplest case with one extra dimension, to a good approximation, the masses of the KK excited gauge bosons are given by $M_{n}=n M_{c}$, where $M_{c}$ is the compactification scale. All KK tower states have identical couplings to the SM fermions. At the LHC, with $100 \mathrm{fb}^{-1}\left(3 \mathrm{ab}^{-1}\right)$, it will be possible to discover 


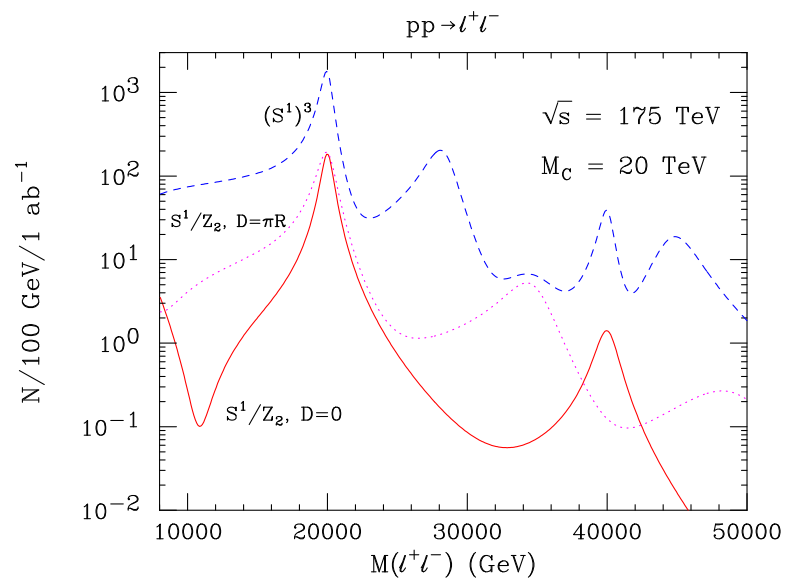

FIG. 21: The number of Drell-Yan events expected per $100 \mathrm{GeV}$ bin for an integrated luminosity of $1 \mathrm{ab}^{-1}$ in several models with two or more extra dimensions and a compactification scale of $M_{c}=20 \mathrm{TeV}$ at the VLHC. Both leptons are required to have rapidity $|\eta(\ell)|<2.5$.

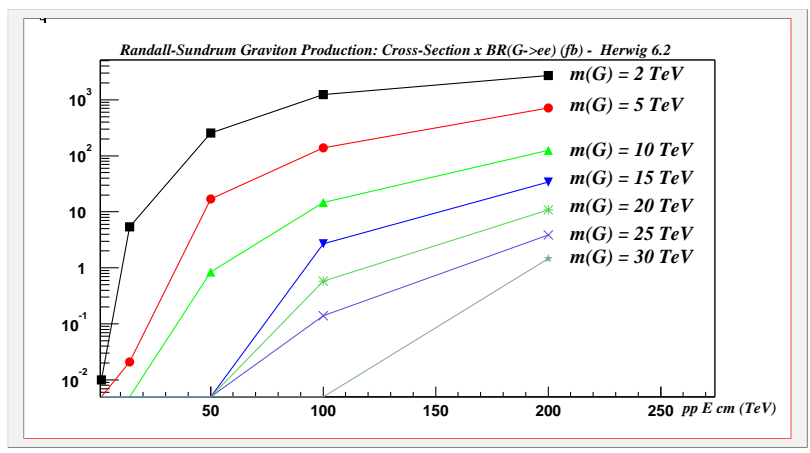

FIG. 22: $\sigma(p p \rightarrow G) \times B R\left(G \rightarrow e^{+} e^{-}\right)$for various graviton masses in models with warped extra dimensions as a function of the center of mass energy for $\Lambda=10 \mathrm{TeV}$.

the lowest lying KK $\gamma, Z$ excitation of this model with mass up to $M_{K K}=5.2 \mathrm{TeV}\left(M_{K K}=6.5 \mathrm{TeV}\right)$ (see Sec. IIE). However, there is no hope of observing higher excitations. At a VLHC with $\sqrt{s}=200 \mathrm{TeV}$ and $100 \mathrm{fb}^{-1}$, the limit can be pushed up to $M_{K K} \approx 30 \mathrm{TeV}$.

For two or more extra dimensions, the masses and couplings of KK excitations become both level and compactification scheme dependent. This leads to a rather complex KK spectrum in processes such as Drell-Yan production. It will be necessary to observe a rather large part of the spectrum in order to experimentally determine the number of extra dimensions and how they are compactified. Some sample KK excitation spectra for a number of different $\mathrm{TeV}$-scale models with more than one extra dimension are shown in Fig. 21. The models are labeled by the manifold on which they are compactified. The spectra in these models are quite distinctive. Measuring the location of the peaks, and their relative heights and widths can be used to uniquely identify a given compactification scheme. Figure 21 suggests that one should be able to differentiate the many possible models for compactification scales up to $M_{c} \approx 20 \mathrm{TeV}$ through detailed cross section measurements.

\section{Warped Extra Dimensions}

In models with warped extra dimensions, one expects to produce $\mathrm{TeV}$-scale graviton resonances in many channels, including the di-lepton channel [78]. In its simplest version, with one extra dimension, two distinct branes, and with all SM fields on the TeV-brane, this model has only two fundamental parameters which can be chosen to be the mass of the first KK state, $m_{1}$, and the scale

$$
\Lambda=\bar{M}_{P l} e^{-k r_{c} \pi},
$$

where $\bar{M}_{P l}$ is the reduced effective 4-D Planck scale, $r_{c}$ is the compactification radius of the extra dimension, and $k$ is a scale of the order of the Planck scale. The masses of the graviton resonances are given by

$$
m_{n}=x_{n}(c) \Lambda
$$

where $c=k / \bar{M}_{P l}$ and $x_{n}$ are the roots of the Bessel functions of order 1, $J_{1}$. The mass of the first excitation is $m_{1} \approx 3.83 \mathrm{c} \Lambda$. The couplings of the massive graviton resonances are given by $1 / \Lambda$. The decay angular distribution in the resonance region can demonstrate that a spin- 2 particle is being produced. Measurements of the relative branching ratios to other clean decay modes such as $G \rightarrow \gamma \gamma$ can prove that one is indeed producing gravitons. Table III lists the branching ratios for a few interesting decays for $m_{1}=2 \mathrm{TeV}$. Detailed studies have shown [79] that, at the LHC with an integrated luminosity of $100 \mathrm{fb}^{-1}$, it will be possible to discover graviton resonances with masses up to $m_{1}=2.1 \mathrm{TeV}$ and to discriminate a spin- 2 from a spin- 1 resonance at 90\% CL for masses up to $1.7 \mathrm{TeV}$. The cross section for the production of the lightest graviton resonance in $p p \rightarrow G \rightarrow e^{+} e^{-}$as a function of $\sqrt{s}$ for $\Lambda=10 \mathrm{TeV}$ and various graviton masses $m(G)=m_{1}$ is shown in Fig. 22. All cross sections were determined using HERWIG6.2 [80], which uses an essentially model independent 
TABLE III: Branching ratios for a $2 \mathrm{TeV}$ graviton resonance for a few interesting decay modes.

\begin{tabular}{|c|c|}
\hline Decay Mode & Branching Ratio $\left(M_{G}=2 \mathrm{TeV}\right)$ \\
\hline$e^{+} e^{-}$ & $2.1 \%$ \\
$\bar{t} t$ & $6.1 \%$ \\
$\gamma \gamma$ & $4.2 \%$ \\
$W^{+} W^{-}$ & $9.0 \%$ \\
$Z Z$ & $4.5 \%$ \\
\hline
\end{tabular}

implementation of the resonance, only depending on the (universal) coupling to the SM fields. At the VLHC-II, with $\sqrt{s}=200 \mathrm{TeV}$ and an integrated luminosity of $1 \mathrm{ab}^{-1}$, it should be possible to observe graviton resonances with masses up to $m_{1} \sim 20 \mathrm{TeV}$.

\section{Black Hole Production at the VLHC}

Should the LHC discover signals of extra dimensions such as KK excitations of gravitons or of the SM gauge bosons, the VLHC might well be able to access mass scales considerably larger than the fundamental (higher dimensional) Planck scale, $M_{P} \sim \mathrm{TeV}$. A particularly exciting consequence of this scenario is the production of black holes (BHs) [81, 82]. Simple estimates 82] of their production cross section, treating the BHs as general relativistic objects, suggest enormous event rates at the VLHC, perhaps as large as $\sim k H z(\sim 1 H z)$ for $M_{B H}=10 \mathrm{TeV}\left(M_{B H}=50 \mathrm{TeV}\right)$.

Once produced, black holes decay primarily via Hawking radiation. The decay of a BH is thermal; it obeys all local conservation laws but otherwise does not discriminate between particle species (of the same mass and spin). BHs thus decay with roughly equal probability to all of the $\approx 60$ particles of the SM. The branching ratio of BHs into leptons thus is about 10\%. Approximately 3\% of its decays result in SM gauge bosons, $5 \%$ in neutrinos, and $2 \%$ in Higgs bosons in the final state. The rest of $\sim 80 \%$ yields hadrons. The number of particles in the final state is typically of $\mathcal{O}(10)$ and increases rapidly with $M_{B H} / M_{P}$. The particles originating from $\mathrm{BH}$ decay each carry an energy of several hundred $\mathrm{GeV}$ on the average. $\mathrm{BH}$ decays resulting in leptons or photons in the final state thus result in clean signals with small SM backgrounds.

Since one expects only about $5 \%$ missing transverse energy per event, it should be possible to estimate $M_{B H}$ from the visible decay products. The Hawking temperature, $T_{H}$, can be determined from the energy spectrum of the final states. One can thus directly test the hypothesis that the observed events originate from BH production and not from other new physics. Furthermore, knowing $T_{H}$ as a function of $M_{B H}$ provides a tool to determine the number of spatial dimensions. $T_{H}$ and $M_{B H}$ are related by

$$
\log \left(T_{H}\right)=-\frac{1}{n+1} \log \left(M_{B H}\right)+\text { const. }
$$

\section{H. Summary of Reach: A VLHC Pocket Guide}

The physics reach of the VLHC is summarized and compared with that of the LHC and a luminosity or energy upgraded LHC in Table IV. The search reach of a SLHC with $1 \mathrm{ab}^{-1}$ is typically $20-30 \%$ higher than that of the LHC. Instead, doubling the energy of the LHC improves the reach by a factor $1.5-2$. In a staged approach to a VLHC, the first stage with $\sqrt{s}=40 \mathrm{TeV}$ would be able to find new particles which are a factor $2-3$ more massive than those which can be accessed at the LHC. At the second stage, with $\sqrt{s}=200 \mathrm{TeV}$, the reach of the LHC can be improved by up to one order of magnitude. Note that Table IV is not exhaustive; only selected cases are shown.

\section{DETECTORS FOR VERY HIGH ENERGY HADRON COLLIDERS}

Given experiences at CERN and Fermilab, a broadly based assault on physics beyond the SM may well still be best probed with "general purpose" $(G P)$ detectors (although a need for specialized devices may indeed grow out of the physics results from the LHC). In the following we discuss general requirements, energy deposition and radiation levels, central tracking and outer muon tracking, and calorimetry for such detectors at the VLHC, 
TABLE IV: Search reach of the LHC, the SLHC and the VLHC for various new physics scenarios.

\begin{tabular}{|c|c|c|c|c|c|}
\hline $\begin{array}{c}\text { physics } \\
\text { scenario }\end{array}$ & $\begin{array}{c}\text { LHC } \\
100 \mathrm{fb}^{-1}\end{array}$ & $\begin{array}{c}\text { SLHC } \\
14 \mathrm{TeV}, 1 \mathrm{ab}^{-1}\end{array}$ & $\begin{array}{c}\text { SLHC } \\
28 \mathrm{TeV}, 100 \mathrm{fb}^{-1}\end{array}$ & $\begin{array}{c}\text { VLHC-I } \\
40 \mathrm{TeV}, 100 \mathrm{fb}^{-1}\end{array}$ & $\begin{array}{c}\text { VLHC-II } \\
200 \mathrm{TeV}^{1} 100 \mathrm{fb}^{-1}\end{array}$ \\
\hline $\bar{t} t H$ coupling & $13 \%$ & $\sim 10 \%$ & $\sim 7 \%$ & $5-10 \%$ & $1-3 \%$ \\
$M_{\bar{g}}, M_{\bar{q}}$ & $2 \mathrm{TeV}$ & $2.5 \mathrm{TeV}$ & $3-4 \mathrm{TeV}$ & $4-5.5 \mathrm{TeV}$ & $\sim 20 \mathrm{TeV}$ \\
mess. field $M_{\Phi}$ & - & - & - & - & $\sim 12 \mathrm{TeV}$ \\
strong $W W$ scat. & $1.7 \sigma$ & $1.6 \sigma$ & $4.5 \sigma$ & $7 \sigma$ & $18 \sigma$ \\
$M_{Z^{\prime}}$ & $4-5 \mathrm{TeV}$ & $5-6 \mathrm{TeV}$ & $7-9 \mathrm{TeV}$ & $10-13 \mathrm{TeV}$ & $30-40 \mathrm{TeV}$ \\
comp. scale $\Lambda$ & $23 \mathrm{TeV}$ & $35 \mathrm{TeV}$ & $35 \mathrm{TeV}$ & $\sim 50 \mathrm{TeV}$ & $\sim 100 \mathrm{TeV}$ \\
$M_{q^{*}}$ & $6.5 \mathrm{TeV}$ & $7.5 \mathrm{TeV}$ & $10 \mathrm{TeV}$ & $13 \mathrm{TeV}$ & $70-75 \mathrm{TeV}$ \\
extra dim., $\delta=2, M_{D}$ & $9 \mathrm{TeV}$ & $12 \mathrm{TeV}$ & $15 \mathrm{TeV}$ & $24 \mathrm{TeV}$ & $65 \mathrm{TeV}$ \\
extra dim., $\delta=4, M_{D}$ & $6 \mathrm{TeV}$ & $7 \mathrm{TeV}$ & $10 \mathrm{TeV}$ & $15 \mathrm{TeV}$ & $45 \mathrm{TeV}$ \\
\hline
\end{tabular}

assuming luminosities in the range $\mathcal{L}=10^{34}-10^{35} \mathrm{~cm}^{-2} \mathrm{~s}^{-1}$. A general conclusion (which deserves further thought) was reached that for a VLHC-I operating at $\sqrt{s}=40-50 \mathrm{TeV}$ and $\mathcal{L}=10^{34} \mathrm{~cm}^{-2} \mathrm{~s}^{-1}$, the technology of the current LHC detectors ATLAS [1] and CMS [2] appears to be sufficient. A secondary conclusion was that, for the machine parameters considered, there were no circumstances in which a GP-like detector could obviously not be built.

The objects to be identified and measured are jets, $\gamma, e, \mu, \tau$, charged tracks, and $\mathbb{H}_{T}$ 44. The $G P$ detectors will probably not be designed with a capability of measuring or identifying individual hadrons. Magnetic analysis is mandatory for charge measurement, momentum analysis, muon identification and $b$-quark measurements. Generic tracking (in front of magnet and calorimetry) is required for $e, \mu, \tau, b$ and may be provided by silicon (or eventually diamond) microstrips, pixels or "3D-pixels". Tagging $b$-jets is important for Higgs boson, top and SUSY physics, which implies micro-vertexing at the $100 \mu \mathrm{m}$ level and the possibility of detecting $e$ and $\mu$ in jets.

Electromagnetic (em) calorimetry is essential for both $e$ - and $\gamma$-identification and measurement as well as measuring well the electromagnetic component of jets; it is likely to have one or more "shower maximum" layers with very high granularity. Hadronic calorimetry is vital for jet spectroscopy $(t \rightarrow j j j, j \ell \nu)$, QCD measurements, as well as many searches for new physics, such as for compositeness, or black hole production. One needs to measure well the direction (the core of the jets) and total energy, as independent as possible of the em:hadronic ratio in the jet. Good hadron calorimetry, with a high dynamic range and good granularity, is also helpful in identifying and measuring (isolated) muons. Aggressive rapidity coverage of the forward hadronic calorimeter is important for jet tagging which plays a crucial role in all weak boson fusion processes (Higgs boson production, weak boson scattering). As shown Sec. II 2 2, a significant fraction of the tagging jets for $\sqrt{s}=200 \mathrm{TeV}$ is produced with rapidities $|\eta(j)|>5$.

Measuring muons is critical. Many massive fundamental objects ( $W, Z, \mathrm{SUSY}, \mathrm{BH})$ decay to muons and one needs to know both their charge and their momentum. In addition, if muons are measured, one can determine $\mathbb{E}_{T}$. The resolution needed is estimated to be $\delta p / p<20 \%$ at $5 \mathrm{TeV}$. The best plan is probably to measure inside the central tracker well, and verify the muonic signature behind the calorimeter with relatively simple tracking. Note that the sagitta of a $5 \mathrm{TeV}$ particle over $2 \mathrm{~m}$ in a $4 \mathrm{~T}$ field is $120 \mu \mathrm{m}$. This may be the driver on the central tracking resolution.

The necessity of measuring all the above objects can be illustrated by the discovery of the top quark by CDF and $\mathrm{D} \varnothing$. Because the top cascades $(t \rightarrow b W, W \rightarrow e \nu, \mu \nu, \tau \nu, j j)$ all the above (except for $\gamma$ and $\tau$ detection) were essential. $\tau$ identification could be important for Higgs physics and testing lepton universality, e.g. in the case of a massive $Z^{\prime}$, and for supersymmetry.

Backgrounds and radiation loads are important for detector design, in particular for luminosities of $\mathcal{L}=$ $10^{35} \mathrm{~cm}^{-2} \mathrm{~s}^{-1}$ or more. As total and inelastic cross sections increase only slowly with energy one can extrapolate with some confidence [83]. Most of the produced hadrons have rather low transverse momentum $\left\langle p_{T}\right\rangle \approx 0.6 \mathrm{GeV}$. The radiation dose in the central region is a function mostly of the luminosity, not the energy. This is not true in the very forward region where particle momenta scale with beam energy. We note that multiplicity distributions also widen only slowly with energy but have long non-Gaussian tails. DPMJET/MARS simulations have been carried out to estimate fluxes of particles 84, 85. At a VLHC-I (VLHC-II) the central tracker will see, at $R=10 \mathrm{~cm}, 3(10) \times 10^{7}$ charged particles per $\mathrm{cm}^{-2} \mathrm{~s}^{-1}$ and a tenth as many neutrons. This corresponds to 10(30) MRad/year. At small polar angles (at an end-cap close to the beam pipe) one expects 100 - 1000 times these fluxes! This is a major issue. Forward muon systems must have well-designed shielding, and then radiation damage could be reduced to an acceptable level. Backgrounds from beam losses are only a few percent 
TABLE V: The average number of underlying events, $\mathcal{N}$, the average number of underlying charged tracks per rapidity unit, $N_{t r}$, and the total transverse energy, $E_{T}^{t o t}$, in a cone of size $\Delta R=0.25$ at the LHC and VLHC-II for several choices of the instantaneous luminosity, $\mathcal{L}$, and the bunch spacing, $\Delta \tau$.

\begin{tabular}{|c|c|c|c|c|c|}
\hline$\sqrt{s}$ & $\mathcal{L}$ & $\Delta \tau$ & $\mathcal{N}$ & $N_{\text {tr }}$ & $E_{T}^{\text {tot }}$ \\
\hline $14 \mathrm{TeV}$ & $10^{34} \mathrm{~cm}^{-2} \mathrm{~s}^{-1}$ & $25 \mathrm{~ns}$ & 20 & $\sim 160$ & $7.6 \mathrm{GeV}$ \\
$14 \mathrm{TeV}$ & $10^{35} \mathrm{~cm}^{-2} \mathrm{~s}^{-1}$ & $12.5 \mathrm{~ns}$ & $100-200$ & $\sim 800-1600$ & $38-76 \mathrm{GeV}$ \\
\hline $200 \mathrm{TeV}$ & $10^{34} \mathrm{~cm}^{-2} \mathrm{~s}^{-1}$ & $18 \mathrm{~ns}$ & 24 & $\sim 240$ & $15 \mathrm{GeV}$ \\
$200 \mathrm{TeV}$ & $10^{34} \mathrm{~cm}^{-2} \mathrm{~s}^{-1}$ & $6 \mathrm{~ns}$ & 8 & $\sim 80$ & $5 \mathrm{GeV}$ \\
$200 \mathrm{TeV}$ & $10^{35} \mathrm{~cm}^{-2} \mathrm{~s}^{-1}$ & $18 \mathrm{~ns}$ & 240 & $\sim 2400$ & $150 \mathrm{GeV}$ \\
$200 \mathrm{TeV}$ & $10^{35} \mathrm{~cm}^{-2} \mathrm{~s}^{-1}$ & $6 \mathrm{~ns}$ & 80 & $\sim 800$ & $50 \mathrm{GeV}$ \\
\hline
\end{tabular}

of backgrounds from collisions, provided one has appropriate shielding [86].

A number of physics processes, such as Higgs boson pair production (see Sec. IIB 5), or the production of messenger fields in gauge mediated supersymmetry breaking models (see Sec. II C 3) would benefit from a luminosity of $\mathcal{L}=10^{35} \mathrm{~cm}^{-2} \mathrm{~s}^{-1}$ or more. Current VLHC machine studies [5] do not exclude this possibility. For such high luminosities, one expects a very large number of interactions per crossing, unless the bunch spacing is greatly reduced compared to that of the LHC. A large number of interactions per crossing leads to a large number of charged tracks and large underlying transverse momenta which make isolation of particles difficult and severely affect jet identification at all but the highest energies. The number of underlying events, together with the number of underlying charged tracks per rapidity unit, and the $E_{T}$ in a cone of size $\Delta R=0.25$ for a number of cases are shown in Table $\mathrm{V}$.

\section{A. Tracking}

Tracking using gaseous drift cells larger than about $1 \mathrm{~cm}$ is too slow for the central tracker, but would be acceptable for the muon system. All silicon central tracking is an option, with strips or ministrips (e.g. $50 \mu \mathrm{m} \times 5 \mathrm{~mm}$ ) or pixels of the pad variety or columns. We consider up to $10^{9}$ elements (40 layers about $4 \mathrm{~m}$ long out to $2 \mathrm{~m}$ radius). In the column or 3D geometry electric field lines end on cylinders with axes normal to the detector plane, so the drift is transverse to the particle direction. One can then have short collection distances and times (most of the signal is induced when the charge is close to the electrode, where the electrode solid angle is large) so the 3D signals are concentrated in time. Keys to the technology are being able to etch deep, near vertical holes and coat them with polysilicon. The first 3D detectors have been successfully fabricated. There is a wide plateau and calculations indicate a pulse duration of about $1 \mathrm{~ns}$. R\&D on these column detectors includes developing techniques for fabricating large areas, minimizing the amount of material for multilayer trackers, and studies of radiation hardness.

\section{B. Calorimetric Techniques}

Both electromagnetic and hadronic calorimeters are essential for measuring electrons, photons and jets. One requires radiation hardness, high granularity, and speed. The different technology choices with their advantages and disadvantages should be clearly spelled out following on experience at Run IIb and LHC. Scintillator read out with embedded wavelength-shifting fibers to a light detector (PMT, APD, HPD) is a well-established technique which may be considered especially for the central region. Lead tungstate $\mathrm{PbWO}_{4}$ or other crystals make a good electromagnetic calorimeter but compromise the jet resolution. Quartz fibers embedded in (e.g.) $\mathrm{Cu}$ gives a low (Cerenkov) signal but are very radiation hard. Silicon pads make a compact calorimeter but radiation hardness is an issue. (CVD) Diamond pads should be radiation hard enough but at present the cost would be prohibitive. This is a clear R\&D issue: to bring down the cost of diamond films so that they can be realistically considered for forward (if not central) calorimetry. High pressure gas calorimetry is cheap, radiation hard and promising, especially for the forward direction. Liquid Argon is intrinsically slow (compared to $18 \mathrm{~ns}$ ) but with signal shaping and leading-edge recognition has been shown to be robust in a relatively high rate environment. A particularly interesting new idea is to use unsegmented dual read-out calorimetry. In this concept, longitudinal quartz fibers, which see mostly the electromagnetic shower, and scintillating fibers, which see mostly the hadronic shower, are embedded in a metal absorber matrix. This can achieve the essential advantages of compensating calorimetry, eliminating the effect of fluctuations in $f_{e m}$. The sampling fraction 
could be as large as needed for optimizing the em response, and the hadronic resolution would not suffer. It is an important R\&D project to construct and test such a dual readout calorimeter.

\section{Muons}

Muons are important for $W, Z, t$ and $b$-physics as well as knowing the missing $E_{T}, \mathbb{F}_{T}$. Because of multiple scattering and energy loss fluctuations in the calorimeter, it is best to measure the muon momentum before the calorimeter, and use the track behind the calorimeter to identify the muon. There are large fluctuations $(20-30 \mathrm{GeV})$ in $p_{\text {out }} / p_{\text {in }}$ for a $1 \mathrm{TeV}$ muon [87]. A goal is to measure the sign of $\approx 10 \mathrm{TeV}$ muons with a combination of field integral $(8 \mathrm{Tm})$, number of points $(40-50)$, and precision per point $(50 \mu \mathrm{m})$. The tracking outside the calorimeter, which is very large in area, can then be relaxed. When muons are isolated one can and should measure any showering losses along their tracks, as these are significant.

\section{Detectors for a VLHC: Findings and Conclusions}

In order to make optimal use of the enormous center of mass energy of a VLHC, hadron calorimeter coverage out to $|\eta|=6-7$ and luminosities in the range $\mathcal{L}=10^{34}-10^{35} \mathrm{~cm}^{-2} \mathrm{~s}^{-1}$ are necessary. At the upper end of this range, numerous technological challenges are present for almost all detector components. From the very preliminary survey d88 described here, one can begin to form tentative conclusions which should lead to specific R\&D efforts. These tentative conclusions are:

1. Early indications suggest that with existing and anticipated technologies, tracking may be manageable at the necessary level. However, a significant amount of R\&D directed towards radiation hard, precise and fast approaches (e.g. silicon pads, 3D pixels) is necessary to conclusively answer this question.

2. It may be possible to identify, sign, and momentum analyze muons at the necessary level for all luminosities. The main issue is the momentum resolution for muons with momenta larger than $1 \mathrm{TeV}$. This needs to be looked at in detail in the context of punch-through and backgrounds.

3. Central calorimetry will likely survive the anticipated radiation doses. However, the large number of interactions per crossing will make jet identification and the measurement of missing transverse momentum difficult, even in the multi hundred GeV region. The alternative of extremely short bunch spacing represents major challenges for triggering and data acquisition.

4. In the forward region, which is important for jet tagging in WBF events, radiation hardness is a major issue. Here, a large scale R\&D effort directed towards quartz fibers, scintillating fibers and silicon/diamond pads is needed in order to see to what rapidities the hadron calorimeter can be extended.

5. A general conclusion reached was that if LHC detectors function as expected at $10^{34} \mathrm{~cm}^{-2} \mathrm{~s}^{-1}$ then scaled detectors with ATLAS/CMS technologies would also work at VLHC-I $(\sqrt{s}=40-50 \mathrm{TeV})$ at the same luminosity.

In an important sense the major R\&D for VLHC detectors is the construction and successful use of LHC detectors ATLAS and CMS. If the LHC detectors were scaled up a factor 1.4 linearly (2.7 in volume) that would be a fair starting point for a VLHC-II at $2 \cdot 10^{34} \mathrm{~cm}^{-2} \mathrm{~s}^{-1}$. But for all detectors we need more radiation hard technology, we need more precision/granularity, and we generally need them to be faster. These are the areas of R\&D needed, together with finding ways of making the detectors cheaper. It is not unreasonable to suppose that we have some 10 years (until $\approx 2010$ ) for the $R \& D$ and then one will have 10 years to build the detectors, for operation $\approx 2020$.

\section{CONCLUSIONS AND OPINIONS}

In this report we have presented preliminary results of a survey of the physics capabilities of a hadron collider operating in the $100 \mathrm{TeV}$ region. We have also studied the detector requirements so that the physics goals can be achieved.

If the Higgs boson is light $\left(M_{H} \leq 200 \mathrm{GeV}\right)$, as indicated by present precision electroweak data, a LC will be an ideal tool to precisely study the properties of such a particle. No such "gold plated" physics case exists at this point for a VLHC. However, although the LHC has excellent prospects of making fundamental discoveries 
beyond the SM, it is unlikely to provide complete answers to all ensuing questions. It is easy to construct scenarios where a convincing reason for building a VLHC emerges. In the following we list some which directly follow from the topics discussed in Sec. III.

- The Tevatron and/or LHC/SLHC finds a Higgs boson and nothing else. A LC will then measure most of the Higgs boson properties if $M_{H}$ is sufficiently small. The VLHC would extend those measurements and be the only device capable of searching for the necessary next scale.

- The Tevatron and/or LHC/SLHC finds a light Higgs boson consistent with a supersymmetric interpretation and nothing else. This could happen for certain points in parameter space of MSUGRA models. In this case the first stage of the VLHC would find supersymmetry.

- The LHC/SLHC finds some supersymmetric particles, but no squarks associated with the first two generations. This situation could easily happen in inverted mass hierarchy models. The VLHC would then find the missing squarks and give important hints which model is realized in nature.

- The LHC/SLHC finds supersymmetry and determines that the messenger scale is of $\mathcal{O}(10-100 \mathrm{TeV})$. In this case, the messenger fields which communicate supersymmetry breaking would be directly produced at the VLHC.

- The LHC/SLHC does not find anything except for a weak indication of an enhanced rate in vector boson scattering at the highest accessible energies. This could happen in certain models where the electroweak symmetry is broken dynamically, such as the K-matrix unitarization scheme. VLHC-I would see a clear signal.

- The LHC/SLHC finds evidence for contact interactions with a scale $\Lambda<60 \mathrm{TeV}$. The VLHC would then be able to directly probe the scale of new physics, eg. in composite models of quarks and leptons, excited quarks would be produced. For such measurements, VLHC-I, II are the obvious devices to exploit that finding.

- The LHC/SLHC finds KK excitations of gravitons and/or the SM gauge bosons. In this case the VLHC could perform detailed measurements of the excitation spectrum. This would make it possible to determine how the additional spatial dimensions associated with the KK excitations are warped or compactified.

- If the LHC/SLHC finds evidence for extra dimensions, the VLHC may well operate sufficiently far above the (higher dimensional) Planck scale such that black holes are produced. BH production would make it possible to actually measure the number of extra dimensions.

In all cases information obtained at the LHC/SLHC gives important clues on what physics lies beyond the SM. However, it is not necessary to wait for results from the LHC in order to start planning for a VLHC now. At some point one, inevitably, will want to explore the multi $10 \mathrm{TeV}$ region. A hadron collider is the only machine we in principal know how to build which can directly discover new physics in this region. With the long lead time for a large project like a hadron collider it is essential to start this process now. Furthermore, this must be done as part of a coordinated and coherent international plan which is part of a comprehensive and global High Energy Physics Program.

At this workshop we have begun to investigate the physics potential of a Very Large Hadron Collider with a center of mass energy up to $200 \mathrm{TeV}$. We have also identified several important areas of detector R\&D for such a machine. In the three weeks available we have barely scratched the surface of many topics. More serious and detailed studies in the next several years are essential to fully unravel the potential of a VLHC.

[1] W. Armstrong et al., "Atlas Technical Proposal", CERN/LHCC-94-43 (1994).

[2] G. Bayatian et al., "The Compact Muons Solenoid Technical Proposal", CERN/LHCC-94-38 (1994).

[3] G. Azuelos et al., ATL-PHYS-2001-002 (March 2001).

[4] G. Ambrosio et al., Fermilab-TM-2149 (June 2001).

[5] S. Peggs et al., "Report of the M4 Working Group on Hadron Colliders", Fermilab-Conf-01/311-E, these proceedings.

[6] V. Krutelyov et al., Phys. Lett. B505, 161 (2001); V. Barger et al., Phys. Lett. B478, 224 (2000).

[7] J. A. Aguilar-Saavedra et al. (ECFA/DESY LC Physics Working Group Collaboration), hep-ph/0106315.

[8] T. Abe et al. (American Linear Collider Working Group Collaboration), SLAC-R-570, Resource book for Snowmass 2001, 30 Jun - 21 Jul 2001, Snowmass, Colorado, hep-ex/0106055, hep-ex/0106056, hep-ex/0106057, and hepex/0106058. 
[9] K. Abe et al. (ACFA Linear Collider Working Group), hep-ph/0109166.

[10] R.W. Assmann et al., CERN Report 2000-008.

[11] M. Battaglia, hep-ph/0103338.

[12] G. Altarelli, L. Conti and V. Lubicz, Phys. Lett. B502, 125 (2001); E. Jenkins, Phys. Rev. D56, 458 (1997); G. Mahlon and S. Parke, Phys. Lett. B347, 394 (1995).

[13] J. Ohnemus and J. F. Owens, Phys. Rev. D43, 3626 (1991); J. Ohnemus, Phys. Rev. D44, 1403 (1991); Phys. Rev. D44, 3477 (1991); Phys. Rev. D47, 940 (1993); U. Baur, T. Han and J. Ohnemus, Phys. Rev. D48, 5140 (1993); Phys. Rev. D51, 3381 (1995); Phys. Rev. D53, 1098 (1996); Phys. Rev. D57, 2823 (1998); B. Mele, P. Nason and G. Ridolfi, Nucl. Phys. B357, 409 (1991); S. Frixione, P. Nason and G. Ridolfi, Nucl. Phys. B383, 3 (1992); S. Frixione, Nucl. Phys. B410, 280 (1993); L. J. Dixon, Z. Kunszt and A. Signer, Phys. Rev. D60, 114037 (1999); D. De Florian and A. Signer, Eur. Phys. J. C16, 105 (2000); J. M. Campbell and R. K. Ellis, Phys. Rev. D60, 113006 (1999).

[14] E. W. Glover and J. J. van der Bij, Nucl. Phys. B 321, 561 (1989); C. Zecher, T. Matsuura and J. J. van der Bij, Z. Phys. C64, 219 (1994); T. Matsuura and J. J. van der Bij, Z. Phys. C51, 259 (1991).

[15] C. Kao and D. A. Dicus, Phys. Rev. D43, 1555 (1991).

[16] M. Carena et al., hep-ph/0010338.

[17] A. Airapetian et al. (ATLAS Collaboration), CERN/LHCC/99-15 (May 1999).

[18] ALEPH, DELPHI, L3, and OPAL Collaborations, LHWG Note/2001-03, hep-ex/0107029.

[19] D. Charlton, talk given at the "International Europhysics Conference on High Physics", Budapest, Hungary, 12 18 July 2001, to appear in the proceedings, hep-ex/0110086; D. Abbaneo et al. (The LEP and SLD Collaborations), hep-ex/01/12021.

[20] D. Denegri et al., hep-ph/0112045 (December 2001).

[21] J.G. Branson et al., hep-ph/0110021.

[22] C. Kao, these proceedings.

[23] M. Hohlfeld, ATL-PHYS-2001-004 (May 2001).

[24] D. Zeppenfeld, R. Kinnunen, A. Nikitenko and E. Richter-Was, Phys. Rev. D62, 013009 (2000).

[25] V. Drollinger, hep-ex/0105017.

[26] A. de Roeck, J. Ellis, and F. Gianotti, hep-ex/0112004 (December 2001).

[27] T. Han and B. McElrath, hep-ph/0201023.

[28] A. Djouadi, W. Kilian, M. Mühlleitner and P. M. Zerwas, Eur. Phys. J. C10, 45 (1999).

[29] For a comparison of the capabilities of the LHC and the LC to determine the Higgs boson parameters, see V. Drollinger and A. Sopczak, CMS NOTE 2000/046.

[30] M. Battaglia and A. de Roeck, hep-ph/0111307, these proceedings.

[31] M. Battaglia, E. Boos, and W. Yao, hep-ph/0111276, these proceedings.

[32] R. Raja et al., hep-ex/0108041.

[33] V. Barger, M. Berger, J. F. Gunion and T. Han, hep-ph/011034, these proceedings.

[34] T. Plehn and D. Rainwater, Phys. Lett. B520, 108 (2001).

[35] R. S. Chivukula and C. Holbling, hep-ph/0110214, these proceedings, and references therein.

[36] T. Plehn, D. Zeppenfeld and S. Su, hep-ph/0110364, these proceedings.

[37] C. Oleari and D. Zeppenfeld, hep-ph/0110338, these proceedings.

[38] A. Belyaev, F. Maltoni and L. Reina, hep-ph/0110274, these proceedings.

[39] F. Maltoni, D. Rainwater and S. Willenbrock, in preparation.

[40] M. Battaglia et al., hep-ph/0106204, CERN-TH/2001-150.

[41] I. Hinchliffe and F.E. Paige, hep-ph/0201141 and these proceedings.

[42] M. Dine, A. Kagan, and S. Samuel, Phys. Lett. B243, 250 (1990).

[43] H. Baer, C. Balazs, P. Mercadante, X. Tata and Y. Wang, Phys. Rev. D63, 015011 (2001).

[44] G. Anderson et al., hep-ph/9710254.

[45] S. Dimopoulos, G.F. Giudice and A. Pomarol, Phys. Lett. B389, 37 (1996); T. Han and R. Hempfling, Phys. Lett. B415, 161 (1997).

[46] T. Han and R.J. Zhang, Phys. Lett. B428, 120 (1998).

[47] I. Hinchliffe and F. Paige, ATL-PHYS-98-134.

[48] U. Baur et al., hep-ph/0111314, these proceedings.

[49] S. Dimopoulos and L. Susskind, Nucl. Phys. B155, 237 (1979); S. Weinberg, Phys. Rev. D19, 1277 (1979).

[50] For a review see, E. H. Simmons, hep-ph/0110196, these proceedings.

[51] C.T. Hill, Phys. Lett. B266, 419 (1990); Phys. Lett. B345, 483 (1995); K. Lane and E. Eichten, Phys. Lett. B352, 382 (1995); K. Lane, Phys. Lett. B433, 96 (1998).

[52] M.S. Chanowitz and M.K. Gaillard, Nucl. Phys. B261, 379 (1985); V. Barger et al., Phys. Rev. D42, 3052 (1990); J. Bagger et al., Phys. Rev. D49, 1246 (1994); Phys. Rev. D52, 3878 (1995).

[53] B.A. Dobrescu and C.T. Hill, Phys. Rev. Lett. 81, 2634 (1998); R.S. Chivukula, B.A. Dobrescu, H. Georgi and C.T. Hill, Phys. Rev. D59, 075003 (1999).

[54] H.J. He, C.T. Hill, and T. Tait, Fermilab-Pub-01/164-T (hep-ph/0108041).

[55] D. Dominici, hep-ph/0110084, these proceedings and references therein.

[56] M. Cvetic and S. Godfrey, hep-ph/9504216, "Electroweak Symmetry Breaking and Beyond the Standard Model" eds. T. Barklow, S. Dawson, H. Haber and J. Siegrist, p. 383 (1995), and references therein.

[57] S. Godfrey, hep-ph/0201093 and these proceedings. 
[58] E. Ma, Phys. Rev. D36, 274 (1987); K.S. Babu et al., Phys. Rev. D36, 878 (1987); J.F. Gunion et al., Int. J. Mod. Phys. A2, 118 (1987); T.G. Rizzo, Phys. Lett. B206, 133 (1988).

[59] H. Georgi, E.E. Jenkins, and E.H. Simmons, Phys. Rev. Lett. 62, 2789 (1989); ibid. 63, 1540(E) (1989); Nucl. Phys. B331, 541 (1990).

[60] F. Abe et al. (CDF Collaboration), Phys. Rev. Lett. 79, 2192 (1997).

[61] V. M. Abazov et al. (DØ Collaboration), Phys. Rev. Lett. 87, 061802 (2001).

[62] E. Eichten, K. Lane, and M. Peskin, Phys. Rev. Lett. 50, 811 (1980).

[63] U. Baur, I. Hinchliffe and D. Zeppenfeld, Int. J. Mod. Phys. A2, 1285 (1987).

[64] U. Baur, M. Spira and P. M. Zerwas, Phys. Rev. D42, 815 (1990).

[65] O. Cakir, C. Leroy and R. Mehdiyev, ATL-PHYS-99-024.

[66] T. Sjostrand, P. Eden, C. Friberg, L. Lönnblad, G. Miu, S. Mrenna and E. Norrbin, Comput. Phys. Commun. 135, 238 (2001).

[67] R. Harris, Fermilab-Conf-96-285-E, Proceedings of the Workshop "New Directions in High Energy Physics", Snowmass, CO, June 25 - July 12, 1996, p. 1010.

[68] O. Cakir and R. Mehdiyev, ATL-PHYS-99-002; O. Cakir, C. Leroy and R. Mehdiyev, ATL-PHYS-99-030.

[69] N. Arkani-Hamed, S. Dimopoulos, and G. Dvali, Phys. Lett. B429, 263 (1998) and Phys. Rev. D59, 086004 (1999); I. Antoniadis, N. Arkani-Hamed, S. Dimopoulos, and G. Dvali, Phys. Lett. B436, 257 (1998).

[70] I. Antoniadis, Phys. Lett. B426, 377 (1990); I. Antoniadis, C. Munoz and M. Quiros, Nucl. Phys. B397, 515 (1993); I. Antoniadis and K. Benalki, Phys. Lett. B326, 69 (1994) and Int. J. Mod. Phys. A15, 4237 (2000); I. Antoniadis, K. Benalki, and M. Quiros, Phys. Lett. B331, 313 (1994).

[71] L. Randall and R. Sundrum, Phys. Rev. Lett. 83, 3370 (1999).

[72] L. Vacavant and I. Hinchliffe, J. Phys. G27, 1839 (2001); hep-ex/0005033 (May 2000).

[73] G.F. Giudice, R. Rattazzi and J.D. Wells, Nucl. Phys. B544, 3 (1999); T. Han, J.D. Lykken and R.J. Zhang, Phys. Rev. D59, 105006 (1999); E.A. Mirabelli, M. Perelstein and M.E. Peskin, Phys. Rev. Lett. 82, 2236 (1999); J.L. Hewett, Phys. Rev. Lett. 82, 4765 (1999); T.G. Rizzo, Phys. Rev. D60, 115010 (1999).

[74] G. Landsberg, these proceedings.

[75] V. Kabachenko, A. Miagkov, and A. Zenin, ATL-PHYS-2001-012.

[76] T.G. Rizzo, hep-ph/0108234, these proceedings.

[77] T.G. Rizzo, hep-ph/0108235, hep-ph/0110202, these proceedings.

[78] H. Davoudiasl, J.L. Hewett, and T.G. Rizzo, Phys. Rev. Lett. 84, 2080 (2000); Phys. Lett. B493, 135 (2000); and Phys. Rev. D63, 075004 (2001).

[79] B. C. Allanach, K. Odagiri, M. A. Parker and B. R. Webber, JHEP 0009, 019 (2000).

[80] G. Corcella, I.G. Knowles, G. Marchesini, S. Moretti, K. Odagiri, P. Richardson, M.H. Seymour and B.R. Webber, JHEP 0101, 010 (2001); hep-ph/0107071.

[81] P.C. Argyres, S. Dimopoulos and J. March-Russell, Phys. Lett. B441, 96 (1998); T. Banks and W. Fischler, hepth/9906038; R. Emparan, G.T. Horowitz,, and R.C. Myers, Phys. Rev. Lett. 85, 499 (2000).

[82] S. Dimopoulos and G. Landsberg, Phys. Rev. Lett. 87, 161602 (2001); S.B. Giddings and S. Thomas, hepph/0106219; S.B. Giddings, hep-ph/0110127 and these proceedings; T.G. Rizzo, hep-ph/0111230 and these proceedings.

[83] W.A. Barletta and H. Leutz, eds., "Supercolliders and Superdetectors", World Scientific, 1993.

[84] S. Rösler, R. Engel and J. Ranft, Proc. Monte Carlo 2000 Conference, Lisbon, Portugal, 23 - 26 October 2000 , Springer, p. 1033.

[85] N. V. Mokhov, "The MARS Code System User's Guide" Fermilab-FN-628 (1995); N. V. Mokhov and O. E. Krivosheev, Proc. Monte Carlo 2000 Conference, Lisbon, Portugal, 23 - 26 October 2000, Springer, p. 943, http://www-ap.fnal.gov/MARS.

[86] V. Strotenko et al., "DØ Shielding Optimization: Simulation and Measurements", DØ Note 3417, 1998, Fermilab.

[87] D. E. Groom et al. (Particle Data Group Collaboration), Eur. Phys. J. C15, 1 (2000).

[88] The talks given in the detector subgroup are available at http://www.pa.msu.edu/ brock/snowmass/literature.html 